\title{
Applying Universal Algebra to Lambda Calculus ${ }^{\star}$
}

\author{
Giulio Manzonetto ${ }^{1}$ and Antonino Salibra ${ }^{1,2} \star \star$ \\ $\{$ gmanzone, salibra\}@dsi.unive.it \\ 1 Laboratoire PPS, CNRS-Université Paris 7 , \\ 2, place Jussieu (case 7014), 75251 Paris Cedex 05, France \\ 2 Università Ca'Foscari di Venezia, \\ Dipartimento di Informatica Via Torino 155, 30172 Venezia, Italy
}

\begin{abstract}
The aim of this paper is double. From one side we survey the knowledge we have acquired these last ten years about the lattice of all $\lambda$-theories (= equational extensions of untyped $\lambda$-calculus) and the models of lambda calculus via universal algebra. This includes positive or negative answers to several questions raised in these years as well as several independent results, the state of the art about the long-standing open questions concerning the representability of $\lambda$-theories as theories of models, and 26 open problems. On the other side, against the common belief, we show that lambda calculus and combinatory logic satisfy interesting algebraic properties. In fact the Stone representation theorem for Boolean algebras can be generalized to combinatory algebras and $\lambda$-abstraction algebras. In every combinatory and $\lambda$-abstraction algebra there is a Boolean algebra of central elements (playing the role of idempotent elements in rings). Central elements are used to represent any combinatory and $\lambda$-abstraction algebra as a weak Boolean product of directly indecomposable algebras (i.e., algebras which cannot be decomposed as the Cartesian product of two other non-trivial algebras). Central elements are also used to provide applications of the representation theorem to lambda calculus. We show that the indecomposable semantics (i.e., the semantics of lambda calculus given in terms of models of lambda calculus, which are directly indecomposable as combinatory algebras) includes the continuous, stable and strongly stable semantics, and the term models of all semisensible $\lambda$-theories. In one of the main results of the paper we show that the indecomposable semantics is equationally incomplete, and this incompleteness is as wide as possible.
\end{abstract}

\section{Introduction}

Among the computational formalisms which have been introduced, the lambda calculus plays an important role as a bridge between logic and computer science. The lambda calculus was originally introduced by Church [?,?] as a foundation for logic, where functions, instead of sets, were primitive, and it turned out to be consistent and successful as a tool for formalizing all computable functions. The rise of computers and the development of programming languages gave a new development to its theoretical studies. The lambda calculus is the kernel of the functional programming paradigm, because its ordinary parameter-binding mechanism corresponds closely to parameter binding in many functional programming languages and to variable binding of quantifiers in logic.

\footnotetext{
* This article is a revised and expanded version of results of the authors presented at the International Conference on Order, Algebra and Logics (Nashville, June 12-16, 2007), and at the 19th Annual IEEE Symposium on Logic in Computer Science (LICS'06) (Seattle, August 12-15, 2006) [?].

** Work partially supported by the Equipe PPS of the University Paris 7-Denis Diderot, and by LIX Laboratoire d'Informatique de l'Ecole Polytechnique (Palaiseau, France).
} 
Lambda calculus has been originally investigated by using mainly syntactical methods (see Barendregt's book [?]). At the beginning researchers have focused their interest on a limited number of equational extensions of lambda calculus, called $\lambda$-theories. They arise by syntactical or semantic considerations. Indeed, a $\lambda$-theory may correspond to a possible operational semantics of lambda calculus, as well as it may be induced by a model of lambda calculus through the kernel congruence relation of the interpretation function. Syntactical proofs of consistency of remarkable $\lambda$-theories (for example, the theory equating all unsolvable $\lambda$-terms) were given in Barendregt's 1971 thesis [?], while one of the most significant $\lambda$-theories is connected with the study of the infinite normal forms of $\lambda$-terms through Böhm trees [?,?]. The set of $\lambda$-theories is naturally equipped with a structure of complete lattice (see [?, Chapter 4]). The bottom element of this lattice is the least $\lambda$-theory $\lambda \beta$, while the top element is the inconsistent $\lambda$-theory. Although researchers have mainly focused their interest on a limited number of them, the lattice of $\lambda$-theories, hereafter denoted by $\lambda T$, has a very rich and complex structure (see e.g. [?,?,?]).

The lambda calculus, although its axioms are all in the form of equations, is not a genuine equational theory since the variable-binding properties of lambda abstraction prevent "variables" in lambda calculus from operating as real algebraic variables. Consequently the general methods that have been developed in universal algebra, for defining the semantics of an arbitrary algebraic theory for instance, are not directly applicable. There have been several attempts to reformulate the lambda calculus as a purely algebraic theory. The earliest, and best known, algebraic models are the combinatory algebras of Curry and Schönfinkel (see [?,?]). Although combinatory algebras do not keep the lambda notation, they have a simple purely equational characterization and were used to provide an intrinsic first-order, but not equational, characterization of the models of lambda calculus, as a special class of combinatory algebras called $\lambda$-models [?, Def. 5.2.7]. The connection between the syntax and the semantics of lambda calculus is established by the completeness theorem of lambda calculus: every $\lambda$-theory is the equational theory of some $\lambda$-model (see [?]).

Semantical methods have been extensively investigated. Topology is at the center of the known approaches to giving models of the untyped lambda calculus. After the first model, found by Scott [?] in 1969 in the category of complete lattices and Scott continuous functions, a large number of mathematical models for lambda calculus have been introduced in various categories of domains and were classified into semantics according to the nature of their representable functions, see e.g. [?,?,?]. Scott continuous semantics [?] is given in the category whose objects are complete partial orders and morphisms are Scott continuous functions. Scott continuous semantics includes the class of graph models, which were isolated in the seventies by Plotkin, Scott and Engeler [?,?,?], and the class of filter models, which were isolated at the beginning of eighties by Barendregt, Coppo and Dezani [?] after the introduction of intersection-type discipline at the end of seventies by Coppo and Dezani [?]. Filter models were investigated by Coppo, Dezani, Barendregt et al. in a series of papers and are perhaps the most established and studied semantics of lambda calculus (see e. g. [?,?,?]). Other semantics of lambda calculus were isolated by Berry [?] and Bucciarelli-Ehrhard [?]: Berry's stable semantics and Bucciarelli-Ehrhard's strongly stable semantics are refinements of the continuous semantics introduced to capture the notion of "sequential" Scott continuous function. All these semantics are structurally and equationally rich $[?, ?, ?]$ in the sense that it is possible to build up $2^{\aleph_{0}} \lambda$-models in each of them inducing, pairwise distinct $\lambda$-theories. Nevertheless, the above denotational semantics do not match all possible operational semantics of lambda calculus. We recall that a semantics of lambda calculus is equationally incomplete if there exists a $\lambda$-theory which is not the theory of any model in the semantics. In the nineties the problem of the equational incompleteness was positively solved 
by Honsell and Ronchi della Rocca [?] for Scott's continuous semantics, and by Bastonero and Gouy for Berry's stable semantics [?]. The proofs of the above results are syntactical and very difficult. In [?,?] it was shown the equational incompleteness of all semantics of lambda calculus that involve monotonicity with respect to some partial order and have a bottom element (including the incompleteness of the strongly stable semantics, which had been conjectured by Bastonero-Gouy and by Berline [?,?]). The proof is simple, general and abstract. First a theorem relating the properties of a graph to the properties of a suitable binary operation on the vertices of the graph is proven. Then the incompleteness is obtained by applying this theorem to the graphs, whose vertices are the elements of a partially ordered model of lambda calculus, and whose edges correspond to the symmetric and antireflexive relation which is the union of the strict order and of the strict dual order of the model. This incompleteness removes the belief that partial orderings with a bottom element are intrinsic to models of the lambda calculus, and that the incompleteness of a semantics is only due to the richness of the structure of representable functions. Instead, the incompleteness is also due to the richness of the structure of $\lambda$-theories.

The need of more abstract and sophisticated mathematical techniques in lambda calculus arises when we recognize the difficulty of the problems we handle, for example in order to investigate the structure of the lattice of $\lambda$-theories (see [?, Chapter 4] and [?,?]) in itself and in connections with the theory of models. Salibra [?,?,?] has launched at the end of the nineties a research program for exploring lambda calculus and combinatory logic using techniques of universal algebra. The remark that the lattice of $\lambda$-theories is isomorphic to the congruence lattice of the term algebra of the least $\lambda$-theory $\lambda \beta$ is the starting point for studying lambda calculus by universal algebraic methods, through the variety generated by the term algebra of $\lambda \beta$. In [?] Salibra has shown that the variety generated by the term algebra of $\lambda \beta$ is axiomatized by the finite schema of identities characterizing $\lambda$-abstraction algebras. The equational theory of $\lambda$-abstraction algebras, introduced by Pigozzi and Salibra [?,?], constitutes a purely algebraic theory of the untyped lambda calculus in the same spirit that cylindric and polyadic (Boolean) algebras constitute an algebraic theory of the first-order predicate logic. The variety LAA of $\lambda$ abstraction algebras is intended as an alternative to the variety CA of combinatory algebras in this regard since it is a first-order algebraic description of lambda calculus, which keeps the lambda notation and hence all the functional intuitions. In [?] Salibra has shown that, for every variety of $\lambda$-abstraction algebras, there exists exactly one $\lambda$-theory whose term algebra generates the variety. Thus, the properties of a $\lambda$-theory can be studied by means of the variety of $\lambda$-abstraction algebras generated by its term algebra.

Long-standing open problems of lambda calculus can be restated in terms of algebraic properties of varieties of $\lambda$-abstraction algebras. For example, the open problem of the order-incompleteness of lambda calculus, raised by Selinger (see [?]), asks for the existence of a $\lambda$-theory not arising as the equational theory of a non-trivially partially ordered model of lambda calculus. A partial answer to the order-incompleteness problem was obtained by Salibra in [?], where it is shown the existence of a $\lambda$-theory not arising as the equational theory of a non-trivially partially ordered model with a finite number of connected components. The order-incompleteness of lambda calculus is equivalent to the existence of an $n$-permutable variety of $\lambda$-abstraction algebras for some natural number $n \geq 2$ (see the remark after Thm. 3.4 in [?]). Plotkin, Selinger and Simpson (see [?]) have shown that 2-permutability and 3-permutability are inconsistent with lambda calculus. The problem of $n$-permutability remains open for $n \geq 4$.

We wonder if it is possible to apply to the varieties LAA and CA the nice results developed in universal algebra in the last thirty years, which essentially connect (a) identities or quasiidentities in the language of lattices satisfied by congruence lattices; (b) properties of the com- 
mutator; and (c) Mal'cev conditions, that characterize properties in varieties by the existence of certain terms involved in certain identities. We recall that the structure of an algebra is affected by the shape of its congruence lattice and that the commutator, a binary operation on this lattice, provides a "measure" of this shape. The commutator was first introduce in group theory, where the concept of Abelian group, and other important concepts, can be defined in terms of the commutator operation on normal subgroups. The extension of the commutator to algebras other than groups is due to the pioneering papers of Smith [?] and Hagemann-Hermann [?]. The commutator is very well behaved in congruence modular varieties (see Freese-McKenzie [?] and Gumm [?]). However, in [?] it was shown that LAA is not congruence modular. As a consequence, it is not possible to apply to LAA the nice theory of commutator developed for congruence modular varieties. Lipparini [?,?] and Kearnes-Szendrei [?] have recently shown that under very weak hypotheses the commutator proves also useful in studying algebras without congruence modularity. However, in [?] Lusin and Salibra have shown that a lattice identity is satisfied by all congruence lattices of $\lambda$-abstraction algebras (combinatory algebras, respectively) iff it is true in all lattices. Thus, there is a common belief that lambda calculus and combinatory logic are algebraically pathological.

On the contrary, we will show that $\lambda$-calculus and combinatory logic $d o$ satisfy interesting algebraic properties. One of the milestones of modern algebra is the Stone representation theorem for Boolean algebras. This result was first generalized by Pierce to commutative rings with unit and next by Comer to the class of algebras with Boolean factor congruences. By applying a theorem by Vaggione [?], we show that Comer's generalization of Stone representation theorem also holds for combinatory and $\lambda$-abstraction algebras: any combinatory (or $\lambda$-abstraction) algebra is isomorphic to a "weak" Boolean product of directly indecomposable algebras (i.e., algebras which cannot be decomposed as the Cartesian product of two other non-trivial algebras). The proof of the representation theorem is based on the fact that every combinatory (or $\lambda$-abstraction) algebra contains a Boolean algebra of central elements (introduced by Vaggione [?] in universal algebra). These elements define a direct decomposition of the algebra as the Cartesian product of two other algebras, just like idempotent elements in rings.

This result suggests a connection between propositional classic logic and combinatory logic; what is the real meaning of this connection remains to be investigated. What we would like to emphasize here is that central elements have been shown fundamental in the application of the representation theorem to $\lambda$-calculus, as it will be explained in the next paragraph.

The representation theorem can be roughly summarized as follows: the directly indecomposable combinatory algebras and $\lambda$-abstraction algebras are the 'building blocks' in the respective varieties. The notion of directly indecomposable combinatory algebra appears to be so relevant that we find it even interesting to speak of the "indecomposable semantics" to denote the class of models of lambda calculus which are directly indecomposable as combinatory algebras. This semantics is very general since, as we will show, it encompasses the continuous, stable and strongly stable semantics, and represents all semisensible $\lambda$-theories (theories which do not equate solvable and unsolvable terms). In one of the main results of the paper we show that the indecomposable semantics, although so general, is (largely) incomplete. More precisely, we will prove that it omits a set of $\lambda$-theories which contains an antichain of cardinality $2^{\aleph_{0}}$ and also countably many intervals of cardinality $2^{\aleph_{0}}$.

In one of the last results of the paper we show that the set of $\lambda$-theories representable in each of the classic semantics of $\lambda$-calculus is not closed under finite intersection, in particular it is not a sublattice of the lattice of all $\lambda$-theories. 
Outline. This paper is organized as follows: In Section 2 we review the basic definitions of universal algebra which are involved in the rest of the paper. Section 3 is devoted to present the $\lambda$-calculus from an algebraic point of view and to recall some results concerning its models. In Section ?? we recall the properties of the lattice of $\lambda$-theories and we provide some new results. The Stone representation theorem for combinatory and $\lambda$-abstraction algebras is presented in Section ??. Section ?? is devoted to the equational incompleteness of the indecomposable semantics. In Section ?? we present 26 open problems concerning models and theories of $\lambda$-calculus.

\section{Preliminaries}

\subsection{Posets}

A poset $\mathcal{S}$ is a pair $(S, \sqsubseteq)$, where $S$ is a set and $\sqsubseteq$ is a reflexive, transitive and antisymmetric relation.

Two elements $s, s^{\prime}$ of a poset are incomparable if neither $s \sqsubseteq s^{\prime}$ nor $s^{\prime} \sqsubseteq s$.

Given a poset $\mathcal{S}$ and $S^{\prime} \subseteq S$ we recall that: $S^{\prime}$ is a chain of $\mathcal{S}$ if it is totally ordered by $\sqsubseteq$, and $S^{\prime}$ is an antichain in case its elements are pairwise incomparable.

The interval notation will have the obvious meaning, e.g., $\mathrm{I}\left[s, s^{\prime}\right]=\left\{s^{\prime \prime} \in S: s \sqsubseteq s^{\prime \prime} \sqsubseteq s^{\prime}\right\}$ and $\mathrm{I}\left[s, s^{\prime}\left[=\mathrm{I}\left[s, s^{\prime}\right]-\left\{s^{\prime}\right\}\right.\right.$.

A subset $X$ of a poset $\mathcal{S}$ is directed if, for all $s, s^{\prime} \in X$, there exists $u \in X$ such that $s \sqsubseteq u$ and $s^{\prime} \sqsubseteq u$.

A poset $\mathcal{S}$ is a complete partial order (cpo, for short) if it has a least element $\perp$, and every directed set $X \subseteq S$ admits a least upper bound $\bigsqcup X$.

An element $s$ of a cpo $\mathcal{S}$ is compact if, for every directed subset $X$ of $\mathcal{S}, s \sqsubseteq \bigsqcup X$ implies $s \sqsubseteq u$ for some $u \in X$.

\subsection{Lattices}

A lattice is a poset $\mathcal{S}=(S, \sqsubseteq)$ such that any two elements $s, s^{\prime} \in S$ have a least upper bound $s \vee s^{\prime}$ and a greatest lower bound $s \wedge s^{\prime}$ which are respectively called, in this context, join and meet. Then, $\sqsubseteq$ is definable from the meet or the join. A lattice is bounded if it has a top $\top$ and a bottom $\perp$ element. A lattice is complete if any $A \subseteq S$ has a least upper bound (then all $A$ 's have also a greatest lower bound); in particular every complete lattice is bounded.

We say that an element $s$ of a bounded lattice $\mathcal{S}$ is an atom (coatom) if it is a minimal element different from $\perp$ (maximal element different from $\top$ ). A lattice $\mathcal{S}$ is atomic (resp. coatomic) if, for every element $s$ of $\mathcal{S}$, there exists an atom $a \leq s$ (resp. $a \geq s$ ).

Two elements $s, s^{\prime}$ of a lattice are incompatible if $s \vee s^{\prime}=\top$; two intervals I $\left[s, s^{\prime}\right], \mathrm{I}\left[s^{\prime \prime}, s^{\prime \prime \prime}\right]$ are incompatible if $s_{1}, s_{2}$ are incompatible, for all $s_{1} \in \mathrm{I}\left[s, s^{\prime}\right]$ and $s_{2} \in \mathrm{I}\left[s^{\prime \prime}, s^{\prime \prime \prime}\right]$.

A lattice $\mathcal{S}$ satisfies the modular law if the following condition holds, for all $a, b, c \in S$ :

$$
a \wedge(b \vee(a \wedge c))=(a \wedge b) \vee(a \wedge c)
$$

\subsection{Algebras}

An algebraic similarity type $\Sigma$ is constituted by a non-empty set of operator symbols together with a function assigning to each operator $f \in \Sigma$ a finite arity. Operator symbols of arity 0 are called nullary operators or constants. 
Definition 1. $A \Sigma$-algebra $\mathbf{A}$ is a tuple $\left(A, f^{\mathbf{A}}\right)_{f \in \Sigma}$, where $A$ is a non-empty set and $f^{\mathbf{A}}: A^{n} \rightarrow$ $A$ is an n-ary function for every $f \in \Sigma$ of arity $n$.

$\mathbf{A}$ is trivial if its underlying set is a singleton.

Definition 2. Given two $\Sigma$-algebras $\mathbf{A}$ and $\mathbf{B}$, a homomorphism from $\mathbf{A}$ into $\mathbf{B}$ is a map $g$ : $A \rightarrow B$ such that $g\left(f^{\mathbf{A}}\left(a_{1}, \ldots, a_{n}\right)\right)=f^{\mathbf{B}}\left(g\left(a_{1}\right), \ldots, g\left(a_{n}\right)\right)$ for each n-ary operation $f \in \Sigma$ and for all $a_{i} \in A$.

Two $\Sigma$-algebras $\mathbf{A}$ and $\mathbf{B}$ are isomorphic, and we write $\mathbf{A} \cong \mathbf{B}$, if there exists a bijective homomorphism from $\mathbf{A}$ into $\mathbf{B}$.

We say that an algebra $\mathbf{A}$ is a reduct of an algebra $\mathbf{B}$ (and that $\mathbf{B}$ is an expansion of $\mathbf{A}$ ) if $\mathbf{A}$ and $\mathbf{B}$ have the same underlying set and $f^{\mathbf{A}}=f^{\mathbf{B}}$ for every operator symbol $f$ in the algebraic similarity type of $\mathbf{A}$. Sometimes we will indicate an expansion of $\mathbf{A}$ as $\left(\mathbf{A}, f_{1}, f_{2}, \ldots\right)$.

\subsection{Congruences}

Given a $\Sigma$-algebra $\mathbf{A}$, a binary relation $\phi$ on $\mathbf{A}$ is compatible if for all $f \in \Sigma$ of arity $n$, and for all $a_{i}, b_{i} \in A$ we have

$$
\left(a_{1}, b_{1}\right) \in \phi, \ldots,\left(a_{n}, b_{n}\right) \in \phi \Rightarrow\left(f^{\mathbf{A}}\left(a_{1}, \ldots, a_{n}\right), f^{\mathbf{A}}\left(b_{1}, \ldots, b_{n}\right)\right) \in \phi .
$$

A compatible equivalence relation on a $\Sigma$-algebra $\mathbf{A}$ is called a congruence. As a matter of notation, we will often write $a \phi b$ or $a={ }_{\phi} b$ for $(a, b) \in \phi$.

Given two congruences $\phi$ and $\psi$ on an algebra $\mathbf{A}$, we can form their relative product:

$$
\psi \circ \phi=\{(a, c):(\exists b \in A) a \phi b \psi c\} .
$$

It is easy to check that $\psi \circ \phi$ is still a compatible relation on $\mathbf{A}$, but not necessarily a congruence.

As a matter of notation, we define $\psi \circ^{1} \phi=\psi$ and $\psi \circ^{n+1} \phi=\psi \circ\left(\phi \circ^{n} \psi\right)$ for $n>0$.

We denote by $\operatorname{Con}(\mathbf{A})$ the complete lattice of the congruences of $\mathbf{A}$, which is a sublattice of the equivalence relations on $\mathbf{A}$. The meet $\phi \wedge \psi$ of two congruences $\phi$ and $\psi$ is their intersection, while their join is the least equivalence relation including $\phi \cup \psi$ :

$$
\phi \vee \psi=\bigcup_{n>0} \phi \circ^{n} \psi
$$

The lattice $\operatorname{Con}(\mathbf{A})$ contains a top and a bottom element:

$$
\nabla^{\mathbf{A}}=A \times A ; \quad \Delta^{\mathbf{A}}=\{(a, a): a \in A\} .
$$

When $\mathbf{A}$ is clear from the context we will omit the superscript $\mathbf{A}$ and write $\nabla, \Delta$.

Lemma 1. Let $\mathbf{A}$ be a $\Sigma$-algebra.

(i) A congruence $\phi \in \operatorname{Con}(\mathbf{A})$ is compact if there exists a finite subset $X$ of $A \times A$ such that $\phi=\theta(X)$.

(ii) If $\nabla^{\mathbf{A}}$ is a compact element, then $\operatorname{Con}(\mathbf{A})$ is a coatomic lattice.

A congruence $\phi$ on $\mathbf{A}$ is called trivial if it is equal to $\nabla$ or $\Delta$.

Notation 1. If $X \subseteq A \times A$ and $\phi$ is a congruence, then we write $\theta_{\phi}(X)$ for the least congruence on $\mathbf{A}$ including $\phi \cup X$. If $\phi=\Delta$, then we write $\theta(X)$ for $\theta_{\Delta}(X)$. $\theta(a, b)$ denotes the congruence $\theta(\{(a, b)\})$.

An algebra $\mathbf{A}$ is simple if $\operatorname{Con}(\mathbf{A})=\{\Delta, \nabla\}$. 


\subsection{Direct and subdirect products}

Given two algebras $\mathbf{A}$ and $\mathbf{B}$, we denote by $\mathbf{A} \times \mathbf{B}$ their (direct) product.

Definition 3. An algebra $\mathbf{A}$ is directly decomposable if there exist two non-trivial algebras $\mathbf{B}$ and $\mathbf{C}$ such that $\mathbf{A} \cong \mathbf{B} \times \mathbf{C}$; otherwise we say that $\mathbf{A}$ is directly indecomposable.

Definition 4. An algebra $\mathbf{A}$ is a subdirect product of the algebras $\left(\mathbf{B}_{i}\right)_{i \in I}$, written $\mathbf{A} \leq \Pi_{i \in I} \mathbf{B}_{i}$, if there exists an embedding $f$ of $\mathbf{A}$ into the direct product $\Pi_{i \in I} \mathbf{B}_{i}$ such that the projection $\pi_{i} \circ f: \mathbf{A} \rightarrow \mathbf{B}_{i}$ is onto for every $i \in I$.

\subsection{Varieties}

Definition 5. A non-empty class $\mathbb{K}$ of algebras of the same similarity type is:

(i) A variety if it is closed under subalgebras, homomorphic images and direct products;

(ii) An equational class if it is axiomatizable by a set of equations.

Birkhoff proved in [?] (see also [?, Thm. 4.131]) that conditions (i) and (ii) are equivalent.

A variety $\mathbb{K}$ of algebras is generated by an algebra $\mathbf{A} \in \mathbb{K}$ if every equation satisfied by $\mathbf{A}$ is also satisfied by every algebra in $\mathbb{K}$. We will denote by $\vee(\mathbf{A})$ the variety generated by $\mathbf{A}$.

Definition 6. A variety $\mathbb{K}$ is a subvariety of a variety $\mathbb{K}^{\prime}$ if $\mathbb{K} \subseteq \mathbb{K}^{\prime}$.

Definition 7. An algebra is

(i) congruence distributive if its congruence lattice $\operatorname{Con}(\mathbf{A})$ is a distributive lattice.

(ii) congruence $n$-permutable $(n \geq 2)$ if $\phi \vee \psi=\phi \circ^{n} \psi$ for all congruences $\phi, \psi \in \operatorname{Con}(\mathbf{A})$.

Definition 8. A variety is

(i) congruence distributive if every algebra in the variety is congruence distributive.

(ii) congruence $n$-permutable ( $n \geq 2)$ if every algebra in the variety is congruence n-permutable.

\subsection{Free algebras}

Let $\mathbb{K}$ be a class of $\Sigma$-algebras and $\mathbf{A}$ be a $\Sigma$-algebra.

Definition 9. If $X$ is a subset of $A$, then we say that $\mathbf{A}$ has the universal mapping property for $\mathbb{K}$ over $X$ if, for every $\mathbf{B} \in \mathbb{K}$ and for every mapping $g: X \rightarrow \mathbf{B}$, there is a unique homomorphism $f: \mathbf{A} \rightarrow \mathbf{B}$ that extends $g$ (i.e., $f(x)=g(x)$ for every $x \in X)$.

Definition 10. $\mathbf{A}$ is free in $\mathbb{K}$ over $X$ if $\mathbf{A} \in \mathbb{K}, \mathbf{A}$ is generated by $X$ and $\mathbf{A}$ has the universal mapping property for $\mathbb{K}$ over $X$.

If $\mathbf{A}$ is free in $\mathbb{K}$ over $X$, then $X$ is called a set of generators for $\mathbf{A}$, and $\mathbf{A}$ is said to be freely generated by $X$.

A free algebra in the class of all $\Sigma$-algebras is called absolutely free. In the following we give a concrete characterization of the absolutely free algebra.

Let $X$ be a set. The set $T_{\Sigma}(X)$ of $\Sigma$-terms over $X$ is defined by induction as follows:

- $x \in T_{\Sigma}(X)$ for every $x \in X$; 
- $a \in T_{\Sigma}(X)$ for every nullary operator $a \in \Sigma$;

- if $t_{1}, \ldots, t_{n} \in T_{\Sigma}(X)(n>1)$ then $f\left(t_{1}, \ldots, t_{n}\right) \in T_{\Sigma}(X)$ for all $f \in \Sigma$ of arity $n$.

A $\Sigma$-term is ground if it does not contain variables. If $t$ is a $\Sigma$-term, we write $t \equiv t\left(x_{1}, \ldots, x_{n}\right)$ if the variables occurring in $t$ are among $x_{1}, \ldots, x_{n}$. If $\mathbf{A}$ is a $\Sigma$-algebra then every $\Sigma$-term $t\left(x_{1}, \ldots, x_{n}\right)$ induces a term operation $t^{\mathbf{A}}: A^{n} \rightarrow A$ defined in the obvious way.

The absolutely free $\Sigma$-algebra over $X$ is the $\Sigma$-algebra

$$
\mathbf{T}_{\boldsymbol{\Sigma}}(X)=\left(T_{\Sigma}(X), f^{T_{\Sigma}(X)}\right)_{f \in \Sigma},
$$

where

$$
f^{T_{\Sigma}(X)}\left(t_{1}, \ldots, t_{n}\right)=f\left(t_{1}, \ldots, t_{n}\right), \quad \text { for all } f \in \Sigma .
$$

We will write $\mathbf{T}_{\boldsymbol{\Sigma}}$ for the absolutely free algebra $\mathbf{T}_{\boldsymbol{\Sigma}}(\emptyset)$ over an empty set of generators.

Definition 11. A $\Sigma$-identity (or $\Sigma$-equation) is a pair $(t, u)$ of $\Sigma$-terms, written also $t=u$. An identity $t\left(x_{1}, \ldots, x_{n}\right)=u\left(x_{1}, \ldots, x_{n}\right)$ holds in a $\Sigma$-algebra $\mathbf{A}$ if the $n$-ary term operations $t^{\mathbf{A}}$ and $u^{\mathbf{A}}$ are equal.

\subsection{Factor congruences}

Definition 12. A congruence $\phi$ on an algebra $\mathbf{A}$ is a factor congruence if there exists another congruence $\bar{\phi}$ such that $\phi \wedge \bar{\phi}=\Delta$ and $\phi \circ \bar{\phi}=\nabla$. In this case we call $(\phi, \bar{\phi})$ a pair of complementary factor congruences.

Under the hypotheses of Definition 12 the homomorphism $f: \mathbf{A} \rightarrow \mathbf{A} / \phi \times \mathbf{A} / \bar{\phi}$ defined by $f(x)=(x / \phi, x / \bar{\phi})$ is an isomorphism. Hence, we have:

Lemma 2. $(\phi, \bar{\phi})$ is a pair of complementary factor congruences of $\mathbf{A}$ if, and only if, $\mathbf{A} \cong$ $\mathbf{A} / \phi \times \mathbf{A} / \bar{\phi}$.

So, the existence of factor congruences is just another way of saying "this algebra is a direct product of simpler algebras".

The set of factor congruences of $\mathbf{A}$ is not, in general, a sublattice of $\operatorname{Con}(\mathbf{A}) . \Delta$ and $\nabla$ are the trivial factor congruences, corresponding to $\mathbf{A} \cong \mathbf{A} \times \mathbf{B}$, where $\mathbf{B}$ is a trivial algebra; of course, $\mathbf{B}$ is isomorphic to $\mathbf{A} / \nabla$ and $\mathbf{A}$ is isomorphic to $\mathbf{A} / \Delta$.

Lemma 3. An algebra $\mathbf{A}$ is directly indecomposable if it admits only the two trivial factor congruences $(\Delta$ and $\nabla)$.

Clearly, every simple algebra is directly indecomposable, while there are algebras which are directly indecomposable but not simple: they have congruences, which however do not split the algebra up neatly as a Cartesian product.

\subsection{Decomposition operators}

Factor congruences can be characterized in terms of certain algebra homomorphisms called decomposition operators (see [?, Def. 4.32] for more details).

Definition 13. A decomposition operation for an algebra $\mathbf{A}$ is a function $f: A \times A \rightarrow A$ such that 
- $f(x, x)=x$

- $f(f(x, y), z)=f(x, z)=f(x, f(y, z))$;

- $f$ is an algebra homomorphism from $\mathbf{A} \times \mathbf{A}$ into $\mathbf{A}$.

There exists a bijective correspondence between pairs of complementary factor congruences and decomposition operations, and thus, between decomposition operations and factorizations like $\mathbf{A} \cong \mathbf{B} \times \mathbf{C}$.

Proposition 1. [?, Thm. 4.33] Given a decomposition operator $f$ the binary relations $\phi$ and $\bar{\phi}$ defined by:

$x \phi y$ if, and only if, $f(x, y)=y$,

$x \bar{\phi} y$ if, and only if, $f(x, y)=x$,

form a pair of complementary factor congruences. Conversely, given a pair $(\phi, \bar{\phi})$ of complementary factor congruences, the map $f$ defined by:

$$
f(x, y)=u \text { if, and only if, } x \phi u \bar{\phi} y,
$$

is a decomposition operation.

Notice that if $(\phi, \bar{\phi})$ is a pair of complementary factor congruences, then for all $x$ and $y$ there is just one element $u$ such that $x \phi u \bar{\phi} y$.

\subsection{Boolean factor congruences and Boolean products}

The product congruence of $\phi_{1} \in \operatorname{Con}(\mathbf{A})$ and $\phi_{2} \in \operatorname{Con}(\mathbf{B})$ is the congruence $\phi_{1} \times \phi_{2}$ on $\mathbf{A} \times \mathbf{B}$ defined by: $(b, c) \phi_{1} \times \phi_{2}\left(b^{\prime}, c^{\prime}\right)$ if, and only if, $b \phi_{1} b^{\prime}$ and $c \phi_{2} c^{\prime}$. We denote by $\operatorname{Con}(\mathbf{A}) \times \operatorname{Con}(\mathbf{B})$ the sublattice of $\operatorname{Con}(\mathbf{A} \times \mathbf{B})$ constituted by all product congruences.

Definition 14. A variety $\mathbb{C}$ of algebras has factorable congruences if for every $\mathbf{A}, \mathbf{B} \in \mathbb{C}$ we have $\operatorname{Con}(\mathbf{A} \times \mathbf{B}) \cong \operatorname{Con}(\mathbf{A}) \times \operatorname{Con}(\mathbf{B})$.

Definition 15. An algebra has Boolean factor congruences if its factor congruences form a Boolean sublattice of the congruence lattice.

Most known examples of varieties in which all algebras have Boolean factor congruences are those with factorable congruences. This is the case, for example, of the congruence distributive varieties, and congruence permutable varieties in which the universal congruences are compact (e.g., the variety of rings with unit).

Lemma 4. (Bigelow-Burris [?, Cor. 1.4]) If a variety $\mathbb{C}$ has factorable congruences, then every $\mathbf{A} \in \mathbb{C}$ has Boolean factor congruences.

The Boolean product construction allows us to transfer numerous fascinating properties of Boolean algebras into other varieties of algebras (see [?, Ch. IV]). Actually, this construction has been presented for several years as "the algebra of global sections of sheaves of algebras over Boolean spaces" (see [?,?]); however, these notions were unnecessarily complex and we prefer to adopt here the following equivalent presentation (see [?]). We recall that a Boolean space is a compact, Hausdorff and totally disconnected topological space. 
Definition 16. A weak Boolean product of a family $(\mathbf{A})_{i \in I}$ of algebras is a subdirect product $\mathbf{A} \leq \Pi_{i \in I} \mathbf{A}_{i}$, where I can be endowed with a Boolean space topology such that:

(i) the set $\left\{i \in I: a_{i}=b_{i}\right\}$ is open for all $a, b \in A$, and

(ii) if $a, b \in A$ and $N$ is a clopen subset of $I$, then the element $c$, defined by $c_{i}=a_{i}$ for every $i \in N$ and $c_{i}=b_{i}$ for every $i \in I-N$, belongs to $A$.

A Boolean product is a weak Boolean product such that the set $\left\{i \in I: a_{i}=b_{i}\right\}$ is clopen (i.e., open and closed) for all $a, b \in A$.

\section{The $\lambda$-calculus in algebraic setting}

The two primitive notions of the untyped $\lambda$-calculus are application, the operation of applying a function to an argument, and lambda abstraction, the process of forming a function from the "expression" defining it.

From now on we consider two fixed countable non-empty sets; namely, the set Na of names, and the set Va of algebraic variables. The elements of $\mathrm{Na}$ will be denoted by $a, b, c, \ldots$, while the elements of Va by $x, y, z, \ldots$

Definition 17. The algebraic similarity type $\Lambda$ of lambda calculus is constituted by a binary operator symbol "."; a nullary operator symbol " $a$ " and a unary operator symbol " $\lambda a$ ", for every $a \in N a$.

The binary operator · is called "application" and the unary operator $\lambda a$ "lambda abstraction".

Definition 18. $A \lambda$-term is a ground $\Lambda$-term.

We recall that the set of all $\Lambda$-terms is denoted by $T_{\Lambda}(\mathrm{Va})$, while the set of $\lambda$-terms by $T_{\Lambda}$. $\Lambda$-terms will be usually denoted by $t, u, v, \ldots$, while $\lambda$-terms by $M, N, P, \ldots$

The following are well known $\lambda$-terms, where the symbol $\equiv$ denotes syntactical equality:

$$
\begin{gathered}
\mathbf{I} \equiv \lambda a(a) ; \quad \mathbf{1} \equiv \lambda a(\lambda b(a \cdot b)) ; \quad \mathbf{T} \equiv \lambda a(\lambda b(a)) ; \quad \mathbf{F} \equiv \lambda a(\lambda b(b)) ; \\
\mathbf{S} \equiv \lambda a(\lambda b(\lambda c((a \cdot c) \cdot(b \cdot c)))) ; \quad \delta \equiv \lambda a(a \cdot a) ; \quad \Omega \equiv \delta \cdot \delta .
\end{gathered}
$$

$\lambda a(a \cdot x)$ is an example of a $\Lambda$-term that is not a $\lambda$-term.

Notation 2. From now on, we will write $\lambda a b c . M$ for $\lambda a(\lambda b(\lambda c(M)))$. The dot "." of the application operator is usually omitted and association is made on the left, so that, for example, $(((a \cdot b) \cdot c) \cdot d) \cdot e$ is written abcde. Then the above $\lambda$-terms can be rewritten as follows:

$$
\begin{gathered}
\mathbf{I} \equiv \lambda a . a ; \quad \mathbf{1} \equiv \lambda a b . a b ; \quad \mathbf{T} \equiv \lambda a b . a ; \quad \mathbf{F} \equiv \lambda a b . b \\
\mathbf{S} \equiv \lambda a b c . a c(b c) ; \quad \delta \equiv \lambda a . a a ; \quad \Omega \equiv \delta \delta .
\end{gathered}
$$

Remark 1. $\Lambda$-terms and algebraic variables are called respectively contexts and holes in Barendregt's book [?, Def. 14.4.1].

An occurrence of a name $a$ in a $\Lambda$-term is bound if it lies within the scope of a lambda abstraction $\lambda a$; otherwise it is called free. For example, the occurrence of $a$ in $\lambda a . a c$ is bound, whilst the one of $c$ is free. The set of free names of $M$ is denoted by $\operatorname{FN}(M)$. A $\lambda$-term without free names is said to be closed. The set of all closed $\lambda$-terms is denoted by $T_{\Lambda}^{o}$. 
Two kinds of substitution In the following we analyze the two kinds of substitutions that are studied in this paper: the substitution for the free occurrences of a name and the substitution for the occurrences of an algebraic variable.

The essential feature of a $\Lambda$-term is that a free name in a $\lambda$-term may become bound when we substitute it for a variable within a $\Lambda$-term. This kind of substitution is the usual one of the equational calculus and it does not matter of free and bound occurrences of names. More precisely, given a $\Lambda$-term $u, t\{x:=u\}$ is defined by induction over the complexity of the $\Lambda$-term $t$ as follows:

$$
\begin{aligned}
& -x\{x:=u\}=u \quad(x \in \mathrm{Va}) \\
& -a\{x:=u\}=a \quad(a \in \mathrm{Na}) \\
& -\left(t \cdot t^{\prime}\right)\{x:=u\}=(t\{x:=u\}) \cdot\left(t^{\prime}\{x:=u\}\right) \\
& -(\lambda a . t)\{x:=u\}=\lambda a . t\{x:=u\} .
\end{aligned}
$$

For example,

$$
(\lambda a . x a)\{x:=\lambda b . a\}=\lambda a .(\lambda b . a) a .
$$

The other substitution is proper of $\lambda$-calculus and concerns $\lambda$-terms. Given a $\lambda$-term $M$, we denote by $M[a:=N]$ the result of substituting the $\lambda$-term $N$ for all free occurrences of $a$ in $M$ subject to the usual proviso about renaming bound names in $M$ to avoid capture of free names in $N$. More precisely $M[a:=N]$ is defined by induction over the complexity of $M$ as follows:

$-a[a:=N]=N$

$-b[a:=N]=b \quad(b \neq a)$

- $(P \cdot Q)[a:=N]=(P[a:=N]) \cdot(Q[a:=N])$

- $(\lambda a . P)[a:=N]=\lambda a . P$

- $b \notin \mathrm{FN}(N) \Rightarrow(\lambda b . P)[a:=N]=\lambda b . P[a:=N] \quad(a \neq b)$

$-b \in \mathrm{FN}(N) \Rightarrow(\lambda b . P)[a:=N]=\lambda c . P[b:=c][a:=N] \quad(a \neq b)$, where $c$ is a new name not occurring neither free nor bound in $P$.

For example,

$$
(\lambda a . b a)[b:=a a]=\lambda c .(a a) c,
$$

where the new name $c$ avoids capture of free names.

Note that the equations between $\lambda$-terms, unlike the associative and commutative laws for example, are not always preserved when arbitrary $\lambda$-terms are substituted for free names (e.g., $\lambda a . b a=\lambda c . b c$ does not imply $\lambda a . c a=\lambda c . c c)$. On the contrary, the equations between $\Lambda$-terms are always preserved when arbitrary $\lambda$-terms are substituted for algebraic variables.

\section{1 $\lambda$-abstraction algebras}

The $\lambda$-theories are the main object of study of the untyped $\lambda$-calculus, when, roughly speaking, we consider "conversion" more important than "reduction".

We start by defining the $\lambda$-theories as congruences including $(\beta)$-conversion (which expresses the way of calculating a function $\lambda a . M$ on an argument $N$ ) and $(\alpha)$-conversion (which avoids capture of free names).

Let us consider now the absolutely free $\Lambda$-algebra $\mathbf{T}_{\boldsymbol{\Lambda}}$ over an empty set of generators (see Section 2.7).

Definition 19. $A \lambda$-theory is any congruence on $\mathbf{T}_{\boldsymbol{\Lambda}}$ including $(\alpha)$ - and $(\beta)$-conversion (here $M, N$ are arbitrary $\lambda$-terms and $a, b$ are names): 
( $\alpha) \lambda a \cdot M=\lambda b \cdot M[a:=b]$, for any name $b$ that does not occur free in $M$,

( $\beta)(\lambda a \cdot M) N=M[a:=N]$.

The least $\lambda$-theory is denoted by $\lambda \beta$, while the quotient of the absolutely free algebra $\mathbf{T}_{\boldsymbol{\Lambda}}$ by a $\lambda$-theory $\phi$ is called the term algebra of $\phi$ and will be denoted by $\mathbf{T}_{\boldsymbol{\Lambda}} / \phi$.

The identities between $\lambda$-terms expressing $(\alpha)$ - and $(\beta)$-conversion do not provide a good algebraization of the untyped $\lambda$-calculus, because algebraic variables do not occur in $\lambda$-terms. In the remaining part of this section we show how it is possible to algebraize the lambda calculus.

The variety $\mathrm{V}\left(\mathbf{T}_{\boldsymbol{\Lambda}} / \lambda \beta\right)$ generated by the term algebra of $\lambda \beta$ is the starting point for studying the lambda calculus by universal algebraic methods.

We recall that, by definition, $\mathrm{V}\left(\mathbf{T}_{\boldsymbol{\Lambda}} / \lambda \beta\right)$ satisfies an identity between $\Lambda$-terms

$$
t\left(x_{1}, \ldots, x_{n}\right)=u\left(x_{1}, \ldots, x_{n}\right)
$$

if, and only if, the term algebra $\mathbf{T}_{\boldsymbol{\Lambda}} / \lambda \beta$ satisfies it. This means that all instances of the above identity, obtained by substituting (without $\alpha$-conversion) $\lambda$-terms for variables in it, fall within $\lambda \beta$ :

$$
t\left(M_{1}, \ldots, M_{n}\right)={ }_{\lambda \beta} u\left(M_{1}, \ldots, M_{n}\right), \quad \text { for all } \lambda \text {-terms } M_{1}, \ldots, M_{n} .
$$

In Theorem 1, which was one of the main results of [?], it is shown that $\mathrm{V}\left(\mathbf{T}_{\boldsymbol{\Lambda}} / \lambda \beta\right)$ is axiomatizable by suitable equations between $\Lambda$-terms characterizing the class of lambda abstraction algebras. Among the seven axioms below, the first six constitute a recursive definition of the abstract substitution operator; they express precisely the meta-mathematical content of $(\beta)$-conversion. The last one is an algebraic translation of $(\alpha)$-conversion.

Definition 20. (Pigozzi and Salibra [?]) A A-algebra

$$
\mathbf{A}=\left(A,{ }^{\cdot}, \lambda a^{\mathbf{A}}, a^{\mathbf{A}}\right)_{a \in N a}
$$

is $a \lambda$-abstraction algebra if it satisfies the following identities, where $a, b, c(a \neq b, b \neq c)$ are names and $x, y, z$ are variables:

$\left(\beta_{1}\right)(\lambda a . a) x=x$

$\left(\beta_{2}\right)(\lambda a . b) x=b$;

$\left(\beta_{3}\right)(\lambda a . x) a=x$

$\left(\beta_{4}\right)(\lambda a a . x) y=\lambda a . x$;

$\left(\beta_{5}\right)(\lambda a . x y) z=(\lambda a . x) z((\lambda a . y) z)$;

$\left(\beta_{6}\right)(\lambda a b . x)((\lambda b . y) c)=\lambda b .(\lambda a . x)((\lambda b . y) c)$;

$(\alpha)(\lambda a .(\lambda b . x) c)=\lambda b .(\lambda a .(\lambda b . x) c) b$.

The class of $\lambda$-abstraction algebras is a variety, denoted by LAA, and therefore it is closed under subalgebras, homomorphic images, and Cartesian products.

In the following theorem it is shown that the term algebras of the $\lambda$-theories are the generators of the subvarieties of LAA.

Theorem 1. (Salibra [?, Thm. 14, 16]) Every variety of $\lambda$-abstraction algebras is generated by the term algebra $\mathbf{T}_{\Lambda} / \phi$ of a suitable $\lambda$-theory $\phi$. In particular,

$$
\mathrm{LAA}=\mathrm{V}\left(\mathbf{T}_{\boldsymbol{\Lambda}} / \lambda \beta\right)
$$


Remark 2. The meta-mathematical content of the phrase "a name $a$ does not occur free in $x$ ", or equivalently " $x$ does not depend on the name $a$ ", can be expressed by an equation:

$$
(\lambda a . x) b=x \quad(b \neq a) .
$$

Then, for example, axiom $\left(\beta_{6}\right)$ assumes the following natural form for all elements $y$ which do not depend on $b$ :

$$
(\lambda a b . x) y=\lambda b .(\lambda a . x) y, \quad(a \neq b) .
$$

Remark 3. Thm. 1 is a consequence of [?, Thm. 13], which is a result relating identities between $\Lambda$-terms and identities between $\lambda$-terms. Let $\mathbf{A}$ be a $\lambda$-abstraction algebra and $t\left(x_{1}, \ldots, x_{n}\right)=$ $u\left(x_{1}, \ldots, x_{n}\right)$ be an identity between $\Lambda$-terms. Then there exist two $\lambda$-terms $M_{t}$ and $M_{u}$ such that

$$
\mathbf{A}=t\left(x_{1}, \ldots, x_{n}\right)=u\left(x_{1}, \ldots, x_{n}\right) \Leftrightarrow \mathbf{A} \models M_{t}=M_{u} .
$$

We remark that the proof of (2) is not trivial, because $\lambda$-abstraction algebras may admit elements which depend on all the names in Na. This is obviously not true for the term algebra of a $\lambda$ theory because every $\lambda$-term is a finite string. As an example of this phenomenon, we consider the Cartesian product $\mathbf{A}=\left(\mathbf{T}_{\boldsymbol{\Lambda}} / \lambda \beta\right)^{\mathrm{Na}}$ of Na-copies of the term algebra of $\lambda \beta$. Then all names in Na occur free in $\left\langle a^{\mathbf{A}}: a \in \mathrm{Na}\right\rangle \in \mathbf{A}$ (see Remark 2). Another example concerns the elements which are free generators of the free LAA-algebra.

What kind of variety is LAA? We wonder if it is possible to apply to $\lambda$-abstraction algebras the nice results developed in universal algebra in the last thirty years. The following theorem seems to show that $\lambda$-calculus is algebraically pathological.

A lattice identity is an identity in the similarity type $\{\vee, \wedge, \perp, \top\}$ of bounded lattices.

Theorem 2. (Lusin-Salibra [?]) Every lattice identity holding in all congruence lattices of algebras in LAA is trivial (i.e., true in all lattices).

Many problems on $\lambda$-calculus may be rephrased as problems of existence of a suitable subvariety of LAA (see Section 1). This explains why it is important to study the structure of the lattice of the subvarieties of LAA, or dually of the lattice of the equational theories of $\lambda$-abstraction algebras. The next theorem shows a first positive algebraic result about the subvarieties of LAA.

Theorem 3. (Berline-Salibra [?]) There exists a congruence distributive variety of $\lambda$-abstraction algebras.

The existence of a variety of $\lambda$-abstraction algebras satisfying strong algebraic properties, such as $n$-permutability or congruence distributivity was an open problem first raised in [?].

\subsection{The models of $\lambda$-calculus}

Combinatory logic is a formalism for writing expressions which denote functions. Combinators are designed to perform the same tasks as $\lambda$-terms, but without using bound names. 
Combinatory algebras. Schönfinkel and Curry discovered that a formal system of combinators, having the same expressive power of the $\lambda$-calculus, can be based on only two primitive combinators.

Definition 21. An algebra $\mathbf{C}=\left(C,{ }^{\mathrm{C}}, \mathbf{k}^{\mathbf{C}}, \mathbf{s}^{\mathbf{C}}\right)$, where $\cdot{ }^{\mathbf{C}}$ is a binary operation and $\mathbf{k}^{\mathbf{C}}, \mathbf{s}^{\mathbf{C}}$ are constants, is called a combinatory algebra (see [?,?]) if it satisfies the following identities:

$$
(\mathbf{k} \cdot x) \cdot y=x ; \quad((\mathbf{s} \cdot x) \cdot y) \cdot z=(x \cdot z) \cdot(y \cdot z) .
$$

The superscript ${ }^{\mathbf{C}}$ and the symbol "." are usually omitted. Association is made on the left, so that, for example, the above axioms can be written as follows:

$$
\mathbf{k} x y=x ; \quad \mathbf{s} x y z=x z(y z) .
$$

The class CA of all combinatory algebras constitutes a variety of algebras and, therefore, it is closed under homomorphic images, subalgebras and direct products.

In the equational language of combinatory algebras the derived combinators $\mathbf{i}, \varepsilon$ and $\varepsilon_{n}$ are defined as follows:

$$
\mathbf{i} \equiv \mathbf{s k k} ; \quad \varepsilon \equiv \varepsilon_{1} \equiv \mathbf{s}(\mathbf{k i}) ; \quad \varepsilon_{n+1} \equiv \mathbf{s}(\mathbf{k} \varepsilon)\left(\mathbf{s}\left(\mathbf{k} \varepsilon_{n}\right)\right) .
$$

Hence, every combinatory algebra satisfies the identities

$$
\mathbf{i} x=x ; \quad \varepsilon x y=x y ; \quad \varepsilon_{2} x y z=x y z ; \quad \varepsilon_{3} x y z u=x y z u .
$$

A function $f: C \rightarrow C$ is representable in a combinatory algebra $\mathbf{C}$ if there exists an element $x \in C$ such that $x \cdot z=f(z)$ for all $z \in C$. In this case, we say that $c$ represents $f$ in $\mathbf{C}$.

Two elements $x, y \in C$ are called extensionally equal if they represent the same function in C. For example, the elements $x$ and $\varepsilon x$ are extensionally equal for every $x \in C$. The combinator $\varepsilon$ will be used in the next subsection to select a canonical representative inside the class of all elements $y$ extensionally equal to a given element $x \in C$.

Lambda Models. Although lambda calculus has been object of study since the early thirties, its model theory developed only much later, following Scott's pioneering model construction. At the end of the seventies, researchers were able to provide a general algebraic characterization of the models of lambda calculus as an elementary subclass of combinatory algebras called $\lambda$-models [?,?].

Definition 22. An environment with values in $C$ is a total function $\rho: N a \rightarrow C$, where Na is the set of names of $\lambda$-calculus.

We denote by $\operatorname{Env}_{C}$ the set of all environments with values in $C$. For every $a \in \mathrm{Na}$ and $x \in C$ we denote by $\rho[a:=x]$ the environment $\rho^{\prime}$ which coincides with $\rho$, except on $a$, where $\rho^{\prime}$ takes the value $x$.

Given a combinatory algebra $\mathbf{C}$, the interpretation of a $\lambda$-term $M$ is a function $|M|: \operatorname{Env}_{C} \rightarrow$ $C$ and it is defined by induction as follows, for every environment $\rho \in \operatorname{Env}_{C}$ :

$$
|a|_{\rho}=\rho(a) ; \quad|M \cdot N|_{\rho}=|M|_{\rho} \cdot|N|_{\rho} ; \quad|\lambda a . M|_{\rho}=\varepsilon \cdot m,
$$

where $m \in C$ is any element representing the following function $f_{a}: C \rightarrow C$ :

$$
f_{a}(x)=|M|_{\rho[a:=x]}, \quad \text { for all } x \in C .
$$


The drawback of the previous definition is that, if $\mathbf{C}$ is an arbitrary combinatory algebra, it may happen that the function $f_{a}$ is not representable in $\mathbf{C}$. The axioms characterizing $\lambda$-models were expressly chosen to make coherent the previous definition of interpretation.

Definition 23. A combinatory algebra $\mathbf{C}$ is called a $\lambda$-model if it satisfies the identities $\varepsilon_{2} \mathbf{k}=\mathbf{k}$, $\varepsilon_{3} \mathbf{s}=\mathbf{s}$ and the Meyer-Scott axiom:

$$
\forall x \forall y(\forall z(x \cdot z=y \cdot z) \Rightarrow \varepsilon \cdot x=\varepsilon \cdot y) .
$$

Here the combinator $\varepsilon$ is used as an inner choice operator. Indeed, given any $x$, the element $\varepsilon \cdot x$ is in the same equivalence class as $x$ w.r.t. extensional equality; and, by Meyer-Scott axiom, $\varepsilon \cdot x=$ $\varepsilon \cdot y$ for every $y$ extensionally equal to $x$. Thus, the set $Y=\left\{x: x \cdot z=f_{a}(z)\right.$ for all $\left.z \in C\right\}$ of elements representing the function $f_{a}$ defined in (??) admits $\varepsilon \cdot m$ as a canonical representative and this does not depend on the choice of $m \in Y$.

As a matter of notation, we write $\mathbf{C}=M=N$ if $|M|_{\rho}=|N|_{\rho}$ for all environments $\rho$. A $\lambda$ model univocally induces a $\lambda$-theory through the kernel congruence relation of the interpretation function.

Definition 24. Let $\mathbf{C}$ be a $\lambda$-model. The equational theory of $\mathbf{C}$ is the $\lambda$-theory defined as follows

$$
\operatorname{Th}(\mathbf{C})=\{(M, N) \in \Lambda \times \Lambda: \mathbf{C} \models M=N\} .
$$

Definition 25. $A \lambda$-model $\mathbf{C}$ represents (or induces) $a \lambda$-theory $\phi$ if $\phi=T h(\mathbf{C})$.

Functional $\lambda$-abstraction algebras and $\lambda$-models. The most natural $\lambda$-abstraction algebras are algebras of functions that are obtained by coordinatizing $\lambda$-models. This situation is analogous to that of algebraic logic: the most natural cylindric (and polyadic) algebras are algebras of functions that are obtained by coordinatizing models of first-order logic.

Definition 26. Let $\mathbf{C}=\left(C,{ }^{\mathbf{C}}, \mathbf{k}^{\mathbf{C}}, \mathbf{s}^{\mathbf{C}}\right)$ be a $\lambda$-model. The high-order expansion

$$
\mathbf{C}^{h}=\left(C^{h},{ }^{h}, \lambda a^{h}, a^{h}\right)_{a \in N a}
$$

of $\mathbf{C}$ is an algebra in the similarity type of $\lambda$-abstraction algebras. The underlying set $C^{h}$ is the set of all functions $F: \operatorname{Env}_{C} \rightarrow C$ satisfying the following condition: for every $\rho \in \operatorname{Env}_{C}$, for every sequence of distinct names $\bar{a}=a_{1} \ldots a_{n}$, there exists an element $u \in C$ (which depends on $F, \rho$ and $\bar{a}$ ) such that, for all $\bar{x}=x_{1} \ldots x_{n} \in C^{n}$,

$$
F(\rho[\bar{a}:=\bar{x}])=u x_{1} \ldots x_{n-1} x_{n} .
$$

The operations of application and lambda abstraction are defined as follows, for all $F, G \in C^{h}$ and $\rho \in \operatorname{Env}_{C}$.

(i) $a^{h}(\rho)=\rho(a)$;

(ii) $\left(F \cdot{ }^{h} G\right)(\rho)=F(\rho) \cdot \mathbf{C} G(\rho)$;

(iii) $\lambda a^{h}(F)(\rho)=\varepsilon \cdot{ }^{\mathbf{C}} x$, where $x \in C$ is any element satisfying $x \cdot{ }^{\mathbf{C}} y=F(\rho[a:=y])$ for all $y \in C$.

The set $C^{h}$ contains the interpretations of all $\lambda$-terms and all constant functions. 
Theorem 4. (Pigozzi-Salibra [?]) The algebra $\mathbf{C}^{h}$ is a $\lambda$-abstraction algebra, and it is the largest algebra of functions $F: \operatorname{Env}_{C} \rightarrow C$ closed under the operations defined in (i)-(iii).

Definition 27. Any algebra isomorphic to a subalgebra of a high-order expansion of a $\lambda$-model is called a functional $\lambda$-abstraction algebra.

The class of all these algebras is denoted by FLA. In [?] it was shown the following representation theorem:

Theorem 5. (Goldblatt-Salibra [?]) LAA = FLA.

In other words, any $\lambda$-abstraction algebra is isomorphic to a subalgebra of a high-order expansion of a suitable $\lambda$-model. This makes clear the connection existing between lambda calculus and combinatory logic.

Infinitary lambda calculus. Various infinitary versions of $\lambda$-calculus have been introduced by several authors in [?,?,?]. Here, as an application of Thm. ??, we recall from [?] the completeness theorem for the infinitary $\lambda$-calculus.

Let $\Lambda_{\perp}$ be the similarity type obtained from the similarity type $\Lambda$ of lambda calculus by adding a new nullary operator symbol $\perp$.

Definition 28. An infinitary $\lambda$-term is a finite or infinite rooted tree such that each leaf is either labeled by a name $a \in N a$ or by the constant $\perp$, and the inner nodes are either binary 'application nodes', or unary 'abstraction nodes', in which case they have a label of the form $\lambda_{a}$ for some $a \in N a$.

The set of infinitary $\lambda$-terms, which contains properly $T_{\Lambda}$, is denoted by $T_{\infty}$ and its elements by $A, B, C, \ldots$

Infinitary $\lambda$-terms arise as 'limits' of infinite sequences of $\beta$-conversions. For example, let $\omega_{3} \equiv \lambda a . a a a$ and $\Omega_{3} \equiv \omega_{3} \omega_{3}$. The $\lambda$-term $\Omega_{3}$ generates an infinite sequence of $\beta$-conversions

$$
\Omega_{3}={ }_{\lambda \beta} \Omega_{3} \omega_{3}={ }_{\lambda \beta}\left(\Omega_{3} \omega_{3}\right) \omega_{3}={ }_{\lambda \beta} \cdots=_{\lambda \beta}\left(\left(\left(\left(\Omega_{3} \omega_{3}\right) \omega_{3}\right) \omega_{3}\right) \omega_{3}\right) \omega_{3}={ }_{\lambda \beta} \cdots .
$$

Then it is natural to consider the infinitary $\lambda$-term

$$
\Omega_{3}^{\infty} \equiv\left(\left(\left(\left(\cdots \omega_{3}\right) \omega_{3}\right) \omega_{3}\right) \omega_{3}\right) \quad \text { with infinitely many } \omega_{3} \text { 's }
$$

as the limit of the above sequence of $\beta$-conversions. $\Omega_{3}^{\infty}$ corresponds to the tree

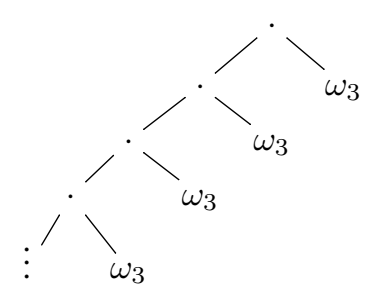

where $\omega_{3}$ is equal to

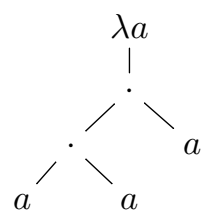

The notions of free and bound occurrence of a name are easily extended to infinitary $\lambda$-terms. The extension of the substitution is more subtle, and sometimes has an unexpected behaviour; we refer the reader to [?, Sec. 3] for more details. Once defined $A[a:=B]$ we can consider the infinitary versions $\left(\alpha^{\infty}\right)$ and $\left(\beta^{\infty}\right)$ of the usual $(\alpha)$ and $(\beta)$-conversions. 
Definition 29. The algebra of infinitary $\lambda$-terms is

$$
\mathbf{T}_{\infty}=\left(T_{\infty}, \cdot{ }^{T_{\infty}}, \lambda_{a}^{T_{\infty}}, a^{T_{\infty}}\right)_{a \in N a},
$$

where $A \cdot{ }^{T} B$ is a tree having an applicative node as root, and $A, B$ as left and right subtrees respectively; $\lambda_{a}^{T_{\infty}}(A)$ is a tree having an abstraction node as root and $A$ as subtree; $a^{T_{\infty}}$ is tree constituted by a unique node labeled by $a$.

Definition 30. An infinitary $\lambda$-theory is any congruence on $\mathbf{T}_{\infty}$ including $\left(\alpha^{\infty}\right)$ - and $\left(\beta^{\infty}\right)$ conversion.

The quotient algebra of $\mathbf{T}_{\infty}$ by an infinitary $\lambda$-theory $\phi$, denoted by $\mathbf{T}_{\infty} / \phi$, is called the term algebra of $\phi$.

Proposition 2. $\mathbf{T}_{\infty} / \phi$ is a $\lambda$-abstraction algebra.

As a consequence of Thm. ?? we have the completeness of the infinitary $\lambda$-calculus.

Theorem 6. (Goldblatt-Salibra [?], Infinitary Completeness Theorem) Let $\phi$ be an infinitary $\lambda$ theory. Then there exists a $\lambda$-model $\mathbf{C}$ such that the term algebra $\mathbf{T}_{\infty} / \phi$ of $\phi$ embeds into $\mathbf{C}^{h}$.

\section{The lattice of $\lambda$-theories}

The interval $\mathrm{I}[\lambda \beta, \nabla]$ of all $\lambda$-theories is a sublattice of the congruence lattice of the absolutely free $\Lambda$-algebra $\mathbf{T}_{\Lambda}$ over an empty set of generators, so that it is isomorphic to the congruence lattice of the term algebra $\mathbf{T}_{\Lambda} / \lambda \beta$ of $\lambda \beta$. The lattice of $\lambda$-theories is naturally equipped with a structure of complete lattice, with meet defined as set-theoretical intersection. The join of two $\lambda$-theories $\phi$ and $\psi$ is the least equivalence relation including $\phi \cup \psi$. It is clear that the bottom element of this lattice is $\lambda \beta$, while the top element $\nabla$ is the inconsistent $\lambda$-theory $\Lambda \times \Lambda$. Although researchers have mainly focused their interest on a limited number of them, the lattice of $\lambda$ theories, hereafter denoted by $\lambda T$, constitutes a very rich and complex structure (see [?,?,?]). Lambda theories interesting for computer scientists can be defined by classifying $\lambda$-terms in terms of their computational behaviour.

Definition 31. A closed $\lambda$-term $M$ is solvable if

$$
M={ }_{\lambda \beta} \lambda a_{1} \ldots a_{n} . a_{i} M_{1} M_{2} \ldots M_{k}, \quad(n, k \geq 0 \text { and } 1 \leq i \leq n)
$$

for some $M_{1}, \ldots, M_{k} \in T_{\Lambda}$. M is unsolvable, otherwise.

Intuitively, solvable $\lambda$-terms are interesting from the computational point of view since they provide at least a partial fixed output, namely $\lambda a_{1} \ldots a_{n} \cdot a_{i}-{ }_{1} \cdots-_{k}$, whilst unsolvable $\lambda$-terms correspond to looping terms.

Looking at the $\lambda$-theories in terms of solvability/unsolvability, they are classified as:

- semisensible if they do not equate a solvable and an unsolvable $\lambda$-term;

- sensible if they equate all unsolvable $\lambda$-terms. 
The following results can be found in [?, Sec. 16, 17]. The $\lambda$-theory $\mathcal{H}$, generated by equating all unsolvable $\lambda$-terms, is the minimal sensible $\lambda$-theory and it is consistent. $\mathcal{H}$ admits a unique maximal consistent extension $\mathcal{H}^{*}$. $\mathcal{H}^{*}$ is a coatom (see Section 2.2) in the lattice of $\lambda$-theories. A $\lambda$-theory $\phi$ is semisensible if, and only if, $\phi \subseteq \mathcal{H}^{*}$ and it is sensible if, and only if, $\mathcal{H} \subseteq \phi$. Sensible consistent $\lambda$-theories are semisensible and never recursively enumerable (r.e., for short).

The semisensible $\lambda$-theory $\lambda \beta \eta$, axiomatized by the axiom of extensionality:

$$
M \cdot a=N \cdot a \Rightarrow M=N, \quad(a \text { not free in } M, N),
$$

does not distinguish $\lambda$-terms which define the same function.

Summarizing, the lattice $\lambda T$ of $\lambda$-theories is divided into two parts: one containing all nonsemisensible $\lambda$-theories and the other one containing all semisensible $\lambda$-theories. The interval $\mathrm{I}\left[\mathcal{H}, \mathcal{H}^{*}\right]$, which belongs to the latter part, constitutes the set of all sensible $\lambda$-theories.

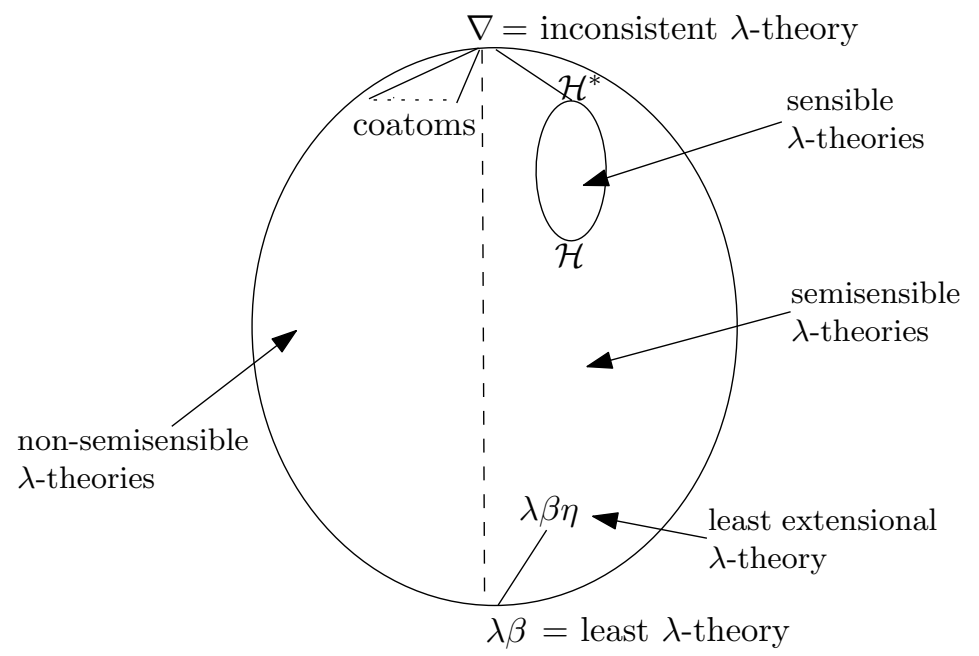

Techniques of universal algebra were applied in [?,?,?] to study the structure of the lattice $\lambda T$ by the variety $\mathrm{V}\left(\mathbf{T}_{\boldsymbol{\Lambda}} / \lambda \beta\right)$ and its subvarieties.

Theorem 7. (Salibra [?]) The lattice of the equational theories of $\lambda$-abstraction algebras is isomorphic to the lattice of $\lambda$-theories.

We summarize in the next theorem some results which enlighten the structure of the lattice of $\lambda$-theories. At the end of the nineties, Salibra proposed the conjecture that the lattice $\lambda T$ satisfies no (non-trivial) lattice identity. This conjecture is still open, because Thm. 2 only implies that every lattice identity $e$ fails in the congruence lattice of a suitable $\lambda$-abstraction algebra that may be different from $\mathbf{T}_{\boldsymbol{\Lambda}} / \lambda \beta$. Moreover, there is a good reason to be also interested in large intervals of the form $\mathrm{I}[\phi, \nabla]$, where $\phi$ is a $\lambda$-theory, because this interval is isomorphic to the congruence lattice of the term algebra of $\phi$, which is a bridge to universal algebra.

Definition 32. Let $\mathcal{S}$ be a bounded lattice with least element $\Delta$ and top element $\nabla$, and $a, b, c_{i}$ $(i \in I)$ be elements of $\mathcal{S}$. We say that $\mathcal{S}$ satisfies the Zipper Condition if the following condition holds:

$$
\text { If } \bigvee_{i \in I} c_{i}=\nabla, c_{i} \wedge a=b(i \in I) \text { then } a=b
$$


The following results have been shown by several authors.

Theorem 8. (i) $\lambda T$ has a continuum of coatoms.

(ii) [?] The meet of all coatoms of $\lambda T$ is different from $\lambda \beta$. In other words, there are identities between non- $(\beta)$-equivalent $\lambda$-terms which are consistent with every $\lambda$-theory.

(iii) [?] Every countable partially ordered set embeds into $\lambda T$ by an order-preserving map.

(iv) [?] Every interval $\mathrm{I}[\phi, \psi]$ where $\phi$ and $\psi$ are r.e. $\lambda$-theories has a continuum of elements.

(v) [?] $\lambda T$ does not satisfy the modular law.

(vi) [?] $\lambda T$ satisfies the Zipper condition.

(vii) [?] There exists a finitely axiomatizable $\lambda$-theory $\phi$ such that the lattice interval $\mathrm{I}[\phi, \nabla]$ is distributive.

Proof. (i) There is a continuum of $\lambda$-theories that are pairwise incompatible (see e.g. [?]).

(ii)-(iv) are shown by using ingenious non-algebraic techniques.

(v) The non-modular pentagon $N_{5}$ (see [?, Thm. 2.25]) embeds into $\lambda T$.

(vi) follows from Thm. ?? and from Lampe's results [?] on the lattices of equational theories (see Thm. ?? below for another proof).

(vii) There is a $\lambda$-theory $\phi$ whose term algebra $\mathbf{T}_{\boldsymbol{\Lambda}} / \phi$ has the lattice operations as term operations.

The remaining results of the section are new.

Let $L$ be a bounded lattice with least element $\Delta$ and top element $\nabla$. For any $x \in L$ we define $L_{x}=\{y \in L-\{\Delta\}: x \wedge y=\Delta\}$. Every element of $L_{x}$ is called a lower semicomplement of $x$. $L$ is said to be lower semicomplemented if $L_{x}$ is non-empty for all $x \neq \nabla$.

Proposition 3. The maximal sensible $\lambda$-theory $\mathcal{H}^{*}$ does not admit a lower semicomplement, so that the lattice of $\lambda$-theories is not lower semicomplemented.

Proof. Let $\phi$ be any non-semisensible $\lambda$-theory. Assume, by the way of contradiction, that $\phi \wedge$ $\mathcal{H}^{*}=\lambda \beta$. It is well known that every equivalence class of a non-semisensible $\lambda$-theory contains an unsolvable $\lambda$-term (see [?]). Then there exists an unsolvable $U$ such that $\mathbf{I}={ }_{\phi} U$. Since for all unsolvable $\lambda$-terms $M$ we have $U M={ }_{\phi} M$ (by $\mathbf{I}={ }_{\phi} U$ ) and $U M={ }_{\mathcal{H}^{*}} M$ (by the sensibility of $\mathcal{H}^{*}$ ), then $U M={ }_{\lambda \beta} M$ follows from $\phi \wedge \mathcal{H}^{*}=\lambda \beta$. In particular, we have $U \Omega=_{\lambda \beta} \Omega$. By [?, Lemma 1.10] this implies that either $U a=_{\lambda \beta} a$ or $U a=_{\lambda \beta} \Omega$, for a new name $a$. In the first case, we contradict the semisensibility of $\lambda \beta$. In the second one we derive $U M={ }_{\lambda \beta} M={ }_{\lambda \beta} \Omega$ for all unsolvable $\lambda$-terms $M$. This contradicts the fact that $\lambda \beta$ is not sensible.

Proposition 4. Let $\phi$ be an r.e. $\lambda$-theory. Then the lattice interval $\mathrm{I}[\phi, \nabla]$ is not lower semicomplemented.

Proof. By [?, Prop. 17.1.9] there exists a $\lambda$-term $M$ such that $\theta_{\phi}(M, N) \neq \nabla$ for all closed $\lambda$-terms $N$. This implies that there exists an infinite number of maximal consistent $\lambda$-theories extending $\phi$. Since the interval $\mathrm{I}[\phi, \nabla]$ is a coatomic complete lattice satisfying the Zipper condition, and admitting a compact top element (see Theorem ??(vi) and Lemma 1), then the conclusion of the proposition follows from [?, Prop. 3], where it is shown that, under the above hypotheses, a lattice is lower semicomplemented if, and only if, the coatoms form a finite decomposition of the least element. 


\subsection{The commutator for $\lambda$-theories}

The structure of an algebra is affected by the shape of its congruence lattice. The commutator, a binary operation on this lattice, provides a "measure" of this shape. In this section we show that the binary commutator on the set of $\lambda$-theories has a good behavior if one of its arguments is $\nabla$. As a consequence, we get that the lattice $\lambda T$ satisfies a condition (in the form of quasi-identity) that, among other things, implies the ET and Zipper conditions.

Given two $\lambda$-theories $\phi$ and $\psi$, we write $\mathcal{X}(\phi, \psi)$ for the set of all $2 \times 2$ matrices $X=X_{i, j}$ $(1 \leq i, j \leq 2)$ of the form:

$$
X=\left(\begin{array}{l}
t\left(\bar{M}_{1}, \bar{N}_{1}\right) t\left(\bar{M}_{1}, \bar{N}_{2}\right) \\
t\left(\bar{M}_{2}, \bar{N}_{1}\right) t\left(\bar{M}_{2}, \bar{N}_{2}\right)
\end{array}\right)
$$

where $\bar{M}_{1}, \bar{M}_{2} \in\left(T_{\Lambda}\right)^{n}, \bar{N}_{1}, \bar{N}_{2} \in\left(T_{\Lambda}\right)^{m}$, for some $n, m \geq 0, t$ is any $m+n$-ary $\Lambda$-term, and $\bar{M}_{1} \phi \bar{M}_{2}, \bar{N}_{1} \psi \bar{N}_{2}$. That is, if in a matrix $X$ we shift along a line then we shift modulus $\psi$, if we shift along a column we shift modulus $\phi$.

If $\tau$ is another $\lambda$-theory, we say that $\phi$ centralizes $\psi \operatorname{modul} \tau$ (see e.g. [?]), in symbols $C(\phi, \psi ; \tau)$, if and only if, for every matrix $X$ such that:

$$
\left(\begin{array}{ll}
M_{1} & N_{1} \\
M_{2} & N_{2}
\end{array}\right) \in \mathcal{X}(\phi, \psi)
$$

we have:

$$
M_{1} \tau N_{1} \Rightarrow M_{2} \tau N_{2} .
$$

The set of all $\lambda$-theories $\tau$ such that $C(\phi, \psi ; \tau)$ is non-empty and closed under arbitrary intersection (see [?]). The commutator $[\phi, \psi]$ of $\phi$ and $\psi$ is defined as the least $\lambda$-theory $\tau$ satisfying $C(\phi, \psi ; \tau)$. Notice that $\phi$ always centralizes $\psi$ modulo $\phi \wedge \psi$, so that we have always $[\phi, \psi] \leq \phi \wedge \psi$.

In this first result we show that the commutator for $\lambda$-theories has a good behavior when one of the involved congruences is $\nabla$.

Theorem 9. Let $\phi$ be a $\lambda$-theory. Then

$$
[\nabla, \phi]=[\phi, \nabla]=\phi .
$$

Proof. Let $M, N$ be $\lambda$-terms such that $M={ }_{\phi} N$. We define:

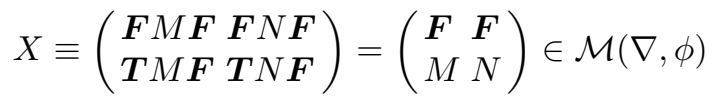

From $(\boldsymbol{F}, \boldsymbol{F}) \in[\nabla, \phi]$ it follows that $(M, N) \in[\nabla, \phi]$. By the arbitrariness of $M$ and $N$ such that $M={ }_{\phi} N$ we obtain that $\phi \leq[\nabla, \phi]$. Since $[\nabla, \phi] \leq \phi$ always holds, we obtain the conclusion. Similarly we can show that $[\phi, \nabla]=\phi$.

Theorem 10. Let $\phi, \psi$ and $\delta_{i}(i \in I)$ be $\lambda$-theories. Then we have:

(i) If $\bigvee_{i \in I} \delta_{i}=\nabla, \phi \geq \psi \wedge\left(\delta_{i} \vee(\phi \wedge \psi)\right)(i \in I)$ then $\psi \leq \phi$.

(ii) If the lattice interval $\mathrm{I}[\psi, \nabla]$ is modular and $\bigvee_{i \in I} \delta_{i}=\nabla$ then $\phi=\bigvee_{i \in I}\left(\delta_{i} \wedge \phi\right)$ for every $\phi, \delta_{i} \geq \psi$. 
Proof. (i) By [?, Prop. 1.2(6)] and by hypothesis we have $C\left(\bigvee_{i \in I} \delta_{i}, \psi ; \phi\right)$. Since $\bigvee_{i \in I} \delta_{i}=$ $\nabla$ and $[\nabla, \psi]=\psi$ we get $\psi \leq \phi$, because the commutator $[\nabla, \psi]$ is the least congruence $\gamma$ satisfying $C(\nabla, \psi ; \gamma)$.

(ii) By [?, Cor. 1.3(e)] and the hypothesis of modularity $\left[\bigvee_{i \in I} \delta_{i}, \phi\right] \leq \bigvee_{i \in I}\left[\delta_{i}, \phi\right]$. Then we have: $\phi=[\nabla, \phi]=\left[\bigvee_{i \in I} \delta_{i}, \phi\right] \leq \bigvee_{i \in I}\left[\delta_{i}, \phi\right] \leq \bigvee_{i \in I} \delta_{i} \wedge \phi$

By putting $\phi=\delta_{i} \wedge \psi$ in Thm. ?? $(i)$ we get another proof of the Zipper condition for $\lambda T$.

\section{The Stone representation theorem for $\lambda$-calculus}

In this section we show that combinatory algebras and $\lambda$-abstraction algebras satisfy a theorem which is similar to the Stone representation theorem for Boolean algebras.

\subsection{The classical Stone and Pierce theorem}

The Stone representation theorem for Boolean rings (the observation that Boolean algebras could be regarded as rings is due to Stone) admits a generalization, due to Pierce, to commutative rings with unit (see [?] and [?, Ch. V]). To help the reader to get familiar with the argument, we now outline Pierce's construction.

Let $\mathbf{A}=(A,+, \cdot, 0,1)$ be a commutative ring with unit, and let $\mathrm{E}(\mathbf{A})=\{a \in A: a \cdot a=a\}$ be the set of its idempotent elements. One defines a structure of Boolean algebra on $\mathrm{E}(\mathbf{A})$ as follows. For all $a, b \in \mathrm{E}(\mathbf{A})$ :

$$
\begin{aligned}
& \text { - } a \wedge b=a \cdot b ; \\
& \text { - } a \vee b=a+b-(a \cdot b) ; \\
& \text { - } a^{-}=1-a .
\end{aligned}
$$

Then it is possible to show that for every $a \in \mathrm{E}(\mathbf{A}), a \neq 0,1$ induces a pair $(\theta(a, 1), \theta(a, 0))$ of non-trivial complementary factor congruences. In other words, the ring $\mathbf{A}$ can be decomposed in a non-trivial way as $\mathbf{A} \cong \mathbf{A} / \theta(a, 1) \times \mathbf{A} / \theta(a, 0)$. If $\mathrm{E}(\mathbf{A})=\{0,1\}$, then $A$ is directly indecomposable. Then Pierce's theorem for commutative rings with unit can be stated as follows:

"Every commutative ring with unit is isomorphic to a Boolean product of directly indecomposable rings."

If $\mathbf{A}$ is a Boolean ring, we get the Stone representation theorem for Boolean algebras, because the ring of truth values is the unique directly indecomposable Boolean ring.

The remaining part of this section is devoted to provide the statement and the proof of the representation theorem for combinatory algebras and $\lambda$-abstraction algebras.

\subsection{The Boolean algebra of central elements}

We start by defining the constants which correspond to the elements 0 and 1 in a commutative ring with unit:

- Combinatory algebras: $1 \equiv \mathbf{k} ; \quad 0 \equiv \mathbf{s k}$,

- $\lambda$-abstraction algebras: $1 \equiv \lambda a b . a ; \quad 0 \equiv \lambda a b . b$. 
As a matter of notation, we set

$$
\theta_{e} \equiv \theta(1, e) ; \quad \bar{\theta}_{e} \equiv \theta(e, 0)
$$

Definition 33. (Vaggione [?,?]) Let $\mathbf{A}$ be an algebra with two constants 0,1 such that $\theta(0,1)=$ $\nabla$. We say that an element e of $\mathbf{A}$ is central, and we write $e \in \operatorname{Ce}(\mathbf{A})$, if $\left(\theta_{e}, \bar{\theta}_{e}\right)$ forms a pair of complementary factor congruences.

A central element e is trivial if it is equal either to 0 or to 1.

Lemma 5. Let $\mathbf{A} \in \mathrm{CA} \cup \mathrm{LAA}$ and $e \in \mathrm{Ce}(\mathbf{A})$. Then we have:

(i) $x \theta_{e}$ exy $\bar{\theta}_{e} y$.

(ii) $x \theta_{e} y$ if, and only if, exy $=y$, and $x \bar{\theta}_{e} y$ if, and only if, exy $=x$.

Proof. $(i)$ Since $1 \theta_{e} e \bar{\theta}_{e} 0$ then $x=1 x y \theta_{e}$ exy $\bar{\theta}_{e} 0 x y=y$.

(ii) By $(i)$.

Lemma 6. Let $\mathbf{A} \in \mathrm{CA} \cup \mathrm{LAA}$ and $e \in A$. Then we have: $e \in \operatorname{Ce}(\mathbf{A})$ if, and only if, $\theta_{e} \wedge \bar{\theta}_{e}=\Delta$.

Proof. $(\Leftarrow) \theta_{e} \circ \bar{\theta}_{e}=\nabla$ follows from Lemma $?$ ? $(i)$.

We now provide a new characterization of the notion of central element which works for combinatory algebras and $\lambda$-abstraction algebras.

Theorem 11. Let $\mathbf{C} \in \mathrm{CA} \cup \mathrm{LAA}$. Then the following conditions are equivalent for all $e \in C$ :

(i) e is central;

(ii) e satisfies the following identities:

1. $e x x=x$,

2. $e(e x y) z=e x z=e x(e y z)$,

3. $e(x y)(z t)=e x z(e y t)$,

4. $e=e 10$,

5. $e(\lambda a . x)(\lambda a . y)=\lambda a . e x y,($ only for LAA).

(iii) The function $f_{e}$ defined by $f_{e}(x, y)=e x y$ is a decomposition operator and $f_{e}(1,0)=e$.

Proof. $($ ii $) \Leftrightarrow($ iii $)$ It is a simple exercise to show that $e$ satisfies the identities in $(i i)$ if, and only if, $f_{e}$ is a decomposition operator such that $f_{e}(0,1)=e$.

$(i) \Rightarrow($ iii $)$ If $e$ is central, then $\left(\theta_{e}, \bar{\theta}_{e}\right)$ is a pair of complementary factor congruences and by Lemma ?? $(i)$ exy is the unique element such that $x \theta_{e}$ exy $\bar{\theta}_{e} y$. It follows that $f_{e}$ is a decomposition operator. Moreover, $f_{e}(1,0)=e 10=e$, because $e$ is the unique element such that $1 \theta_{e} e \bar{\theta}_{e} 0$.

$($ iii $) \Rightarrow(i)$ Let $(\phi, \bar{\phi})$ be the pair of complementary factor congruences associated with $f_{e}$, that is, $x \phi y$ iff $e x y=x$, and $x \bar{\phi} y$ iff $e x y=y$. We recall that $e x y$ is the unique element such that $x \phi \operatorname{exy} \bar{\phi} y$. Since $f_{e}(1,0)=e$ then $e$ is the unique element such that $1 \phi e \bar{\phi} 0$. It follows that $\theta_{e} \subseteq \phi$ and $\bar{\theta}_{e} \subseteq \bar{\phi}$. For the opposite direction, let $x \phi y$, i.e., exy $=x$. Then, by $1 \theta_{e} e$ we have $x=1 x y \theta_{e}$ exy $=y$. Similarly, for $\bar{\phi}$. $\{1,0\}$.

It follows that an algebra $\mathbf{C} \in \mathrm{CA} \cup \mathrm{LAA}$ is directly indecomposable if, and only if, $\mathrm{Ce}(\mathbf{C})=$ 
Theorem 12. Let $\operatorname{Dec}(\mathbf{C})$ be the set of decomposition operators of an algebra $\mathbf{C} \in \mathrm{CA} \cup \mathrm{LAA}$. Then the functions, mapping central elements into decomposition operators

$$
e \in \mathrm{Ce}(\mathbf{C}) \rightarrow f_{e}, \quad \text { where } f_{e}(x, y)=e x y
$$

and decomposition operators into central elements

$$
f \in \operatorname{Dec}(\mathbf{C}) \rightarrow f(1,0) \in \operatorname{Ce}(\mathbf{C}),
$$

form the two sides of a bijection.

Proof. Let $f$ be a decomposition operator and let $e=f(1,0)$. We now show that $e$ is central and that $f(x, y)=e x y$. The element $e$ is the unique one satisfying $1 \phi e \bar{\phi} 0$, where $(\phi, \bar{\phi})$ is the pair of complementary factor congruences associated with the decomposition operator $f$. Since $\phi$ and $\bar{\phi}$ are compatible equivalence relations, it follows that for all $x, y$ :

$$
x=1 x y \phi \operatorname{exy} \bar{\phi} 0 x y=y .
$$

Since, by definition, $f(x, y)$ is the unique element satisfying $x \phi f(x, y) \bar{\phi} y$, we obtain:

$$
f(x, y)=e x y .
$$

Finally, the identities defining $f$ as decomposition operator make $e$ a central element by Thm. ??

We now check that these correspondences form the two sides of a bijection. Assume $e$ is central, that is $\left(\theta_{e}, \bar{\theta}_{e}\right)$ is a pair of complementary factor congruences. Then $f(x, y)=e x y$ is a decomposition operator because $x \theta_{e}$ exy $\bar{\theta}_{e} y$. If $f$ is a decomposition operator, then by (??) we have that $f_{f(1,0)}(x, y)=f(1,0) x y=f(x, y)$ for all $x, y$.

Corollary 1. The functions, mapping central elements into pairs of complementary factor congruences

$$
e \in \mathrm{Ce}(\mathbf{C}) \rightarrow\left(\theta_{e}, \bar{\theta}_{e}\right),
$$

and pairs of complementary factor congruences into central elements

$$
(\phi, \bar{\phi}) \rightarrow e \quad \text { if } 1 \phi e \bar{\phi} 0,
$$

form the two sides of a bijection.

Lemma 7. The varieties CA and LAA have factorable congruences. Hence, every algebra $\mathbf{C} \in$ $\mathrm{CA} \cup \mathrm{LAA}$ has Boolean factor congruences.

Proof. Let A, B be combinatory algebras or $\lambda$-abstraction algebras; it is clear that, up to isomorphism, $\operatorname{Con}(\mathbf{A}) \times \operatorname{Con}(\mathbf{B}) \subseteq \operatorname{Con}(\mathbf{A} \times \mathbf{B})$. Conversely, let $\phi \in \operatorname{Con}(\mathbf{A} \times \mathbf{B})$. The "projections" $\phi_{1}, \phi_{2}$ of $\phi$ are the binary relations on $\mathbf{A}$ and $\mathbf{B}$, respectively, defined as follows:

$$
\begin{aligned}
& x_{1} \phi_{1} x_{2} \Longleftrightarrow \exists y_{1}, y_{2} \in B \text { such that }\left(x_{1}, y_{1}\right) \phi\left(x_{2}, y_{2}\right), \\
& y_{1} \phi_{2} y_{2} \Longleftrightarrow \exists x_{1}, x_{2} \in A \text { such that }\left(x_{1}, y_{1}\right) \phi\left(x_{2}, y_{2}\right) .
\end{aligned}
$$

It is obvious that $\phi \subseteq \phi_{1} \times \phi_{2}$. We now prove the opposite inclusion. Suppose that $\left(x_{1}, y_{1}\right) \phi_{1} \times$ $\phi_{2}\left(x_{2}, y_{2}\right)$ for some $x_{1}, x_{2} \in A$ and $y_{1}, y_{2} \in B$. Then, by definition of $\phi_{1} \times \phi_{2}$, we have that 
$x_{1} \phi_{1} x_{2}$ and $y_{1} \phi_{2} y_{2}$. Hence, there exist $x_{3}, x_{4} \in A, y_{3}, y_{4} \in B$ such that $\left(x_{1}, y_{3}\right) \phi\left(x_{2}, y_{4}\right)$ and $\left(x_{3}, y_{1}\right) \phi\left(x_{4}, y_{2}\right)$. Since $(1,0) \phi(1,0)$ and $\phi$ is a compatible relation, we get:

$$
\left(x_{1}, y_{1}\right)=\left(1 x_{1} x_{3}, 0 y_{3} y_{1}\right) \phi\left(1 x_{2} x_{4}, 0 y_{4} y_{2}\right)=\left(x_{2}, y_{2}\right) .
$$

Thus we get $\phi=\phi_{1} \times \phi_{2}$. It is easy to check that $\phi_{1}, \phi_{2}$ are reflexive, symmetric and compatible. We now show that $\phi_{1}$ is also transitive. Let $x_{1} \phi_{1} x_{2} \phi_{1} x_{3}$, then there exist $y_{1}, y_{2}, y_{3}, y_{4}$ such that $\left(x_{1}, y_{1}\right) \phi\left(x_{2}, y_{2}\right)$ and $\left(x_{2}, y_{3}\right) \phi\left(x_{3}, y_{4}\right)$; from the symmetry of $\phi$ we have also $\left(x_{3}, y_{4}\right) \phi\left(x_{2}, y_{3}\right)$. Since $(1,0) \phi(1,0)$ and $\phi$ is a compatible relation, we get:

$$
\left(x_{1}, y_{4}\right)=\left(1 x_{1} x_{3}, 0 y_{1} y_{4}\right) \phi\left(1 x_{2} x_{2}, 0 y_{2} y_{3}\right)=\left(x_{2}, y_{3}\right) .
$$

Finally, from $\left(x_{1}, y_{4}\right) \phi\left(x_{2}, y_{3}\right)$ and $\left(x_{2}, y_{3}\right) \phi\left(x_{3}, y_{4}\right)$ we get $\left(x_{1}, y_{4}\right) \phi\left(x_{3}, y_{4}\right)$ and, hence, $x_{1} \phi_{1} x_{3}$; thus $\phi_{1} \in \operatorname{Con}(\mathbf{A})$. An analogous reasoning gives $\phi_{2} \in \operatorname{Con}(\mathbf{B})$. From this it is easy to conclude that $\operatorname{Con}(\mathbf{A} \times \mathbf{B}) \cong \operatorname{Con}(\mathbf{A}) \times \operatorname{Con}(\mathbf{B})$. By Lemma 4 , every algebra of a variety with factorable congruences has Boolean factor congruences.

We now show that the partial ordering between central elements, defined by:

$$
x \leq y \text { if, and only if, } \bar{\theta}_{x} \subseteq \bar{\theta}_{y}
$$

is a Boolean ordering and the meet, join and complementation operations are internally representable. 0 and 1 are respectively the bottom element and the top element of this ordering.

Theorem 13. The algebra $(\mathrm{Ce}(\mathbf{C}), \wedge, \vee,-, 0,1)$ of central elements of $\mathbf{C}$, defined by

$$
x \wedge y=x y 0 ; \quad x \vee y=x 1 y ; \quad x^{-}=x 01,
$$

is a Boolean algebra isomorphic to the Boolean algebra of factor congruences.

Proof. By Lemma ?? C has Boolean factor congruences. It follows that the partial ordering on central elements, defined in (??), is a Boolean ordering. There only remains to show that, for all central elements $x, y$, the elements $x^{-}, x \wedge y$ and $x \vee y$ are central and are respectively associated with the pairs $\left(\bar{\theta}_{x}, \theta_{x}\right),\left(\theta_{x} \vee \theta_{y}, \bar{\theta}_{x} \wedge \bar{\theta}_{x}\right)$ and $\left(\theta_{x} \wedge \theta_{y}, \bar{\theta}_{x} \vee \bar{\theta}_{x}\right)$ of complementary factor congruences.

We check the details for $x^{-}$. Since $x$ is central then $\left(\theta_{x}, \bar{\theta}_{x}\right)$ is a pair of complementary factor congruences. The complement is the pair $\left(\bar{\theta}_{x}, \theta_{x}\right)$. We have that $x^{-}$is the unique element such that $0 \theta_{x} x^{-} \bar{\theta}_{x}$. Then $1 \bar{\theta}_{x} x^{-} \theta_{x} 0$ for the pair $\left(\bar{\theta}_{x}, \theta_{x}\right)$. This means that $x^{-}$is the central element associated with the pair $\left(\bar{\theta}_{x}, \theta_{x}\right)$.

We now consider $x \vee y=x 1 y$. First of all, we show that $x 1 y=y 1 x$. By Lemma ?? $(i)$ we have that $1 \theta_{x} x 1 y \bar{\theta}_{x} y$, while $1 \theta_{x} y 1 x \bar{\theta}_{x} y$ can be obtained as follows:

$$
\begin{aligned}
& 1=y 11 \text { by Thm. ??(ii-1), } \\
& y 11 \theta_{x} y 1 x \text { by } 1 \theta_{x} x, \\
& y 1 x \bar{\theta}_{x} y 10 \text { by } x \bar{\theta}_{x} 0, \\
& y 10=y \quad \text { by Thm. ??(ii-4). }
\end{aligned}
$$

Since there is a unique element $c$ such that $1 \theta_{x} c \bar{\theta}_{x} y$, then we have the conclusion $x 1 y=y 1 x$. We now show that $x 1 y$ is the central element associated with the factor congruence $\theta_{x} \wedge \theta_{y}$, i.e.,

$$
1\left(\theta_{e} \wedge \theta_{d}\right) x 1 y\left(\bar{\theta}_{e} \vee \bar{\theta}_{d}\right) 0 .
$$

From $y 1 x=x 1 y$ we easily get that $1 \theta_{x} x 1 y$ and $1 \theta_{d} x 1 y$, that is, $1\left(\theta_{e} \wedge \theta_{d}\right) x 1 y$. Finally, by Lemma ??, we have: $x 1 y \bar{\theta}_{e} y=y 10 \bar{\theta}_{d}$ 0, i.e., $x 1 y\left(\bar{\theta}_{e} \vee \bar{\theta}_{d}\right) 0$. The same reasoning works for $x \wedge y$. 
We now provide the promised representation theorem. If $I$ is a maximal ideal of the Boolean algebra $\mathrm{Ce}(\mathbf{A})$, then $\theta_{I}$ denotes the congruence on $\mathbf{A}$ defined by:

$$
x\left(\theta_{I}\right) y \text { if, and only if, } x \theta_{e} y \text { for some } e \in I .
$$

By a Pierce variety (see [?] for the general definition) we mean here a variety of algebras for which there are two constants 0,1 and a term $u(x, y, z, v)$ such that the following identities hold: $u(x, y, 0,1)=x$ and $u(x, y, 1,0)=y$.

Obviously, the variety of combinatory algebras and that of $\lambda$-abstraction algebras are Pierce varieties: in both cases it is sufficient to take $u(x, y, z, v) \equiv z y x$.

Theorem 14. (Representation Theorem for CA and LAA) Let $\mathbf{C} \in \mathrm{CA} \cup \mathrm{LAA}$ and $X$ be the Boolean space of maximal ideals of the Boolean algebra of central elements. Then, for all $I \in X$ the quotient algebra $\mathbf{C} / \theta_{I}$ is directly indecomposable and the map

$$
f: C \rightarrow \Pi_{I \in X}\left(C / \theta_{I}\right),
$$

defined by

$$
f(x)=\left(x / \theta_{I}: I \in X\right),
$$

gives a weak Boolean product representation of $\mathbf{C}$.

Proof. By Lemma ?? the factor congruences of $\mathbf{C}$ constitute a Boolean sublattice of $\operatorname{Con}(\mathbf{C})$. Then by [?] $f$ gives a weak Boolean product representation of $\mathbf{C}$. The quotient algebras $\mathbf{C} / \theta_{I}$ are directly indecomposable by [?, Thm. 8], because the varieties CA and LAA are Pierce varieties.

Note that, in general, it is not possible to obtain a (non-weak) Boolean product representation of an algebra $\mathbf{C} \in \mathrm{CA} \cup$ LAA. This follows from Lemma ?? and two results due to Vaggione [?] and Plotkin-Simpson [?]. Vaggione has shown that, if a variety has factorable congruences and every member of the variety can be represented as a Boolean product of directly indecomposable algebras, then the variety is a discriminator variety (see [?] for the terminology). Discriminator varieties satisfy very strong algebraic properties, in particular they are congruence permutable (i.e., in each algebra the join of two congruences is just their composition). Plotkin and Simpson [?, Thm. 3.7] have shown that this last property is inconsistent with $\lambda$-calculus and combinatory logic, hence by Lemma ?? and Vaggione's theorem not all combinatory algebras and $\lambda$-abstraction algebras have a Boolean product representation.

\section{The indecomposable semantics}

The Stone representation theorem for combinatory algebras can be roughly summarized as follows: the directly indecomposable combinatory algebras are the "building blocks" in the variety of combinatory algebras. Then it is natural to investigate the class of models of $\lambda$-calculus, which are directly indecomposable as combinatory algebras (indecomposable semantics, for short).

In this section we show that the indecomposable semantics encompasses the Scott-continuous, the stable and the strongly stable semantics, and represents all semisensible $\lambda$-theories. In spite of this richness, in the last results of this chapter we show that the indecomposable semantics is incomplete, and that this incompleteness is as wide as possible. Finally, we will show that the set of $\lambda$-theories induced by each of the main semantics is not closed under finite intersection, and hence it does not form a sublattice of $\lambda T$. 


\subsection{The main semantics of $\lambda$-calculus}

After Scott, several models of $\lambda$-calculus have been defined by order theoretic methods and classified into "semantics" according to the nature of their representable functions (see [?], for a survey on these semantics).

The Scott-continuous semantics corresponds to the class of $\lambda$-models having cpo's (complete partial orders) as underlying sets and representing all Scott continuous functions.

The stable semantics (Berry [?]) and the strongly stable semantics (Bucciarelli-Ehrhard [?]) are refinements of the Scott-continuous semantics which have been introduced to capture the notion of "sequential" continuous function. The underlying sets of the $\lambda$-models living in the stable (strongly stable) semantics are particular algebraic cpo's called dI-domains (dI-domains with coherences). These models represent all stable (strongly stable) functions between such domains. A function between dI-domains is stable if it is continuous and, furthermore, commutes with "infs of compatible elements". A strongly stable function between dI-domains with coherence, is a stable function preserving coherence. We refer the reader to [?,?] for a more detailed description of these semantics.

All these semantics are structurally and equationally rich: in particular, in each of them it is possible to build up $2^{\aleph_{0}}$ models having pairwise distinct, and even incompatible, $\lambda$-theories.

\subsection{Incompleteness of the indecomposable semantics}

We now define various notions of representability of $\lambda$-theories in classes of models.

Definition 34. Given a class $\mathbb{C}$ of $\lambda$-models and a $\lambda$-theory $\phi$, we say that:

1. $\mathbb{C}$ represents $\phi$ if there is some $\mathbf{C} \in \mathbb{C}$ representing $\phi$ (i.e., $T h(\mathbf{C})=\phi$ ).

2. $\mathbb{C}$ omits $\phi$ if there is no $\mathbf{C} \in \mathbb{C}$ representing $\phi$ (i.e., $\operatorname{Th}(\mathbf{C}) \neq \phi$ for all $\mathbf{C} \in \mathbb{C}$ ).

3. $\mathbb{C}$ is complete for a set $S \subseteq \lambda T$ of $\lambda$-theories if $\mathbb{C}$ represents all elements of $S$ (i.e., for every $\phi \in S$ there exists $\mathbf{C} \in \mathbb{C}$ such that $T h(\mathbf{C})=\phi$ ).

4. $\mathbb{C}$ is incomplete if it omits a consistent $\lambda$-theory (i.e., there exists $\phi \in S$ such that $T h(\mathbf{C}) \neq \phi$ for all $\mathbf{C} \in \mathbb{C}$ ).

We now remark that the class of directly indecomposable combinatory algebras is a universal class (i.e., it is an elementary class which can be axiomatized by universal sentences).

Proposition 5. The class of all directly indecomposable combinatory algebras ( $\lambda$-abstraction algebras, respectively) is a universal class, so that it is closed under subalgebras and ultraproducts.

Proof. By [?, Prop. 1.3].

The closure of the class of directly indecomposable combinatory algebras under subalgebras is the key trick in the proof of the algebraic incompleteness theorem.

We have shown that any factor congruence can be represented by a central element, and in particular that a combinatory (or $\lambda$-abstraction) algebra $\mathbf{C}$ is directly indecomposable if, and only if, it only admits the trivial central elements.

If $\mathbf{A}$ is a $\lambda$-abstraction algebra, the combinatory reduct of $\mathbf{A}$ is the algebra

$$
\operatorname{Cr}(\mathbf{A})=\left(A,{ }^{\mathbf{A}},(\lambda a b \cdot a)^{\mathbf{A}},(\lambda a b c \cdot a c(b c))^{\mathbf{A}}\right) .
$$


By [?, Thm. 29] $\mathrm{Cr}(\mathbf{A})$ is always a combinatory algebra. By [?, Cor. 5.2.13(ii)] it is a $\lambda$-model under the hypothesis that $\mathbf{A}$ is the term algebra of a $\lambda$-theory.

In every $\lambda$-model the interpretations of the combinators $\mathbf{k}$ and sk coincide with those of the $\lambda$-terms $\lambda a b . a$ and $\lambda a b . b$. Then the role of the trivial central elements in a $\lambda$-abstraction algebra and in its combinatory reduct is covered by the same elements.

Lemma 8. Let $\phi$ be a $\lambda$-theory and $M$ be a closed $\lambda$-term. If $[M]_{\phi}$ is a non-trivial central element of the term algebra $\boldsymbol{\Lambda}_{\phi}$, then every $\lambda$-model representing $\phi$ is directly decomposable. It follows that the indecomposable semantics omits $\phi$.

Proof. Let $\mathbf{C}$ be a $\lambda$-model. Then $T h(\mathbf{C})=\phi$ if, and only if, $\boldsymbol{\Lambda}_{\phi}$ is isomorphic to a subalgebra of the $\lambda$-abstraction expansion $\mathbf{C}^{\lambda}$ of $\mathbf{C}$ (see Section ??). By Prop. ?? and by the hypothesis we obtain that $\mathbf{C}^{\lambda}$ is decomposable. Then the combinatory reduct $\operatorname{Cr}\left(\mathbf{C}^{\lambda}\right)$ is decomposable. Finally, $\mathbf{C}$ is decomposable because it is a subalgebra of $\operatorname{Cr}\left(\mathbf{C}^{\lambda}\right)$ that contains the interpretation of all closed terms.

We are now able to provide the promised algebraic incompleteness theorem.

Theorem 15. (Algebraic incompleteness theorem) The indecomposable semantics is incomplete.

Proof. By Lemma ?? it is sufficient to produce a $\lambda$-theory $\phi$ such that the term algebra $\boldsymbol{\Lambda}_{\phi}$ of $\phi$ has a non-trivial central element. By [?, Prop. 15.3.9] the $\lambda$-theories $\theta(\Omega, \lambda a b . a)$ and $\theta(\Omega, \lambda a b . b)$ are non-trivial. Then, we conclude by Lemma ?? that $[\Omega]_{\phi}$, where $\phi=\theta(\Omega, \lambda a b . a) \wedge \theta(\Omega, \lambda a b . b)$, is a non-trivial central element of $\boldsymbol{\Lambda}_{\phi}$.

\subsection{Continuous, stable and strongly stable semantics}

In Thm. ?? below we show that, although the indecomposable semantics is incomplete, it is large enough to represent all semisensible $\lambda$-theories.

We need now a technical lemma.

Lemma 9. Let $\phi$ be a $\lambda$-theory and e be a non-trivial central element of $\boldsymbol{\Lambda}_{\phi}$. Then, every $\lambda$-term belonging to the equivalence class e is unsolvable.

Proof. Let $M \in e$. Since the congruences $\theta_{e}$ and $\bar{\theta}_{e}$ on $\boldsymbol{\Lambda}_{\phi}$ are non-trivial, then the $\lambda$-theories $\phi_{1}=\theta_{\phi}(\lambda a b . b, M)$ and $\phi_{2}=\theta_{\phi}(\lambda a b . a, M)$ are consistent. By [?, Lemma 10.4.1(i)] it is consistent to equate two solvable $\lambda$-terms only if they are equivalent according to [?, Def. 10.2.9]. If $M$ were solvable then it should be equivalent both to $\lambda a b . b$ and $\lambda a b . a$, so that these last terms should be equivalent. But this is false. Then $M$ must be unsolvable.

Theorem 16. The indecomposable semantics represents all semisensible $\lambda$-theories.

Proof. Let $\phi$ be a semisensible $\lambda$-theory. Assume, by the way of contradiction, that $\boldsymbol{\Lambda}_{\phi}$ has a nontrivial central element $e$ (cf. Lemma ??). Let $M \in e$. Then, $\boldsymbol{\Lambda}_{\phi}$ satisfies the identity exx $=x$ from which we derive $M P P=P$ for every solvable $P$. This contradicts the semisensibility of $\phi$ since $M$ is unsolvable by Lemma??.

In the next proposition we show that all $\lambda$-models living in the main semantics are simple algebras. We recall that an algebra is simple when it has just the two trivial congruences, and is hence directly indecomposable. 


\section{Proposition 6.}

(i) All $\lambda$-models living in the Scott-continuous semantics are simple combinatory algebras.

(ii) All $\lambda$-models living in the stable or strongly stable semantics are simple combinatory algebras.

Proof. Let us consider a $\lambda$-model $\mathbf{C}=(\mathcal{D}, \cdot, \mathbf{k}, \mathbf{s})$.

(i) Suppose that $\mathbf{C}$ lives in Scott-continuous semantics, so that $\mathcal{D}$ is a cpo and all Scott continuous functions are representable in $\mathbf{C}$. It is easy to check that, for all $b, c \in \mathcal{D}$, the function $g_{b, c}$ defined by

$$
g_{b, c}(x)= \begin{cases}c & \text { if } x \nsubseteq \mathcal{D} b, \\ \perp & \text { otherwise },\end{cases}
$$

is Scott continuous. Let $\phi$ be a congruence on $\mathbf{C}$ and suppose that there exist $a, d$ such that $a \phi d$ with $a \neq d$. We have $a \square_{\mathcal{D}} d$ or $d \Xi_{\mathcal{D}} a$. Suppose, without loss of generality, that we are in the first case. Since the continuous function $g_{d, c}$ is representable in the model (for all $c$ ), we have: $\perp=g_{d, c}(a) \phi g_{d, c}(d)=c$, hence $c \phi \perp$. By the arbitrariness of $c$ we get that $\phi$ is trivial, so that $\mathbf{C}$ is simple. Note that $g_{d, c}$ is neither stable nor strongly stable hence it cannot be used for proving item $($ ii).

(ii) Suppose that $\mathbf{C}$ is a (strongly) stable $\lambda$-model. Consider two elements $a, b \in \mathcal{D}$ such that $a \neq b$. We have $a \square_{\mathcal{D}} b$ or $b \square_{\mathcal{D}} a$. Suppose, without loss of generality, that we are in the first case. Then there is a compact element $d$ of $\mathbf{C}$ such that $d \sqsubseteq_{\mathcal{D}} a$ and $d \Xi_{\mathcal{D}} b$. The step function $f_{d, c}$ defined by :

$$
f_{d, c}(x)= \begin{cases}c & \text { if } d \sqsubseteq \mathcal{D} x, \\ \perp & \text { otherwise, }\end{cases}
$$

is stable (strongly stable) for every element $c$. This function $f_{d, c}$ can be used to show that every congruence on $\mathbf{C}$ is trivial as in the proof of item $(i)$.

As a consequence of Prop. ??, we get, in a uniform way, the incompleteness of the main semantics of $\lambda$-calculus. We will see later on that this incompleteness is very large.

Corollary 2. The Scott-continuous, the stable and the strongly stable semantics are incomplete.

Proof. By Prop. ?? and Thm. ??.

Given a class $\mathbb{C}$ of $\lambda$-models, $T h(\mathbb{C})$ denotes the set of $\lambda$-theories which are represented in $\mathbb{C}$. In the remaining part of this subsection we show that, for each of the classic semantics of $\lambda$-calculus, the set $T h(\mathbb{C})$ is not closed under finite intersection, so that it is not a sublattice of the lattice $\lambda T$ of $\lambda$-theories.

Theorem 17. Let $\mathbb{C}$ be a class of directly indecomposable models of $\lambda$-calculus. If there are two consistent $\lambda$-theories $\phi, \psi \in T h(\mathbb{C})$ such that

$$
\Omega={ }_{\phi} \lambda a b . a ; \quad \Omega={ }_{\psi} \lambda a b . b,
$$

then $T h(\mathbb{C})$ is not closed under finite intersection, so it is not a sublattice of $\lambda T$.

Proof. Let $\xi=\phi \wedge \psi$. By Lemma ??, $[\Omega]_{\xi}$ is a non-trivial central element of $\boldsymbol{\Lambda}_{\xi}$. It follows that $\xi \notin T h(\mathbb{C})$. 
We recall that the graph models (see, e.g., [?,?]) and the filter models (see, e.g., [?]) are classes of $\lambda$-models within the Scott-continuous semantics.

Corollary 3. Let $\mathbb{C}$ be one of the following semantics: graph semantics, filter semantics, Scottcontinuous semantics, stable semantics, strongly stable semantics. Then $T h(\mathbb{C})$ is not a sublattice of $\lambda T$.

Proof. In each of these semantics it has been proved that for all $M \in \Lambda^{o}$ there exists a model C such that $T h(\mathbf{C})$ is consistent and $\Omega={ }_{T h(\mathbf{C})} M$. Then the conclusion follows from Thm. ?? and Prop. ??.

\subsection{Concerning the number of decomposable and indecomposable $\lambda$-models}

From the work done in the previous subsection, it is easy to conclude that there is a wealth of directly indecomposable $\lambda$-models representing different $\lambda$-theories.

Theorem 18. Let $\mathbb{C}_{\mathbb{I N D}}$ be the indecomposable semantics. Then $T h\left(\mathbb{C}_{\mathbb{I N D}}\right)$ contains an interval of cardinality $2^{\aleph_{0}}$ and an antichain of cardinality $2^{\aleph_{0}}$.

Proof. We know from Thm. ?? that $T h\left(\mathbb{C}_{\mathbb{I N D}}\right)$ contains the interval $\mathrm{I}\left[\lambda \beta, \mathcal{H}^{*}\right]$, which has cardinality $2^{\aleph_{0}}$ by [?, Sec. 16.3] (see Section ?? for the definition of $\mathcal{H}^{*}$ ). Moreover, Cor. ?? implies that $T h\left(\mathbb{C}_{\mathbb{I N D}}\right)$ also contains the set of all $\lambda$-theories represented by the class of graph models, which has an antichain of cardinality $2^{\aleph_{0}}$ by [?].

Now, we show that also the incompleteness of the indecomposable semantics is as wide as possible.

First of all we need some results about $\lambda$-theories. The proof of the following lemma is similar to that of [?, Prop. 17.1.9], where the case $k=1$ (due to Visser) is shown, and it is omitted.

Lemma 10. Suppose that $\phi$ is an r.e. $\lambda$-theory and fix arbitrary pairs of $\lambda$-terms $\left(M_{i}, N_{i}\right)$ for $1 \leq i \leq k$ such that $M_{i} \neq_{\phi} N_{i}$ for all $i \leq k$. Then there is $M \in \Lambda^{o}$ such that, for all $P \in \Lambda^{\circ}$, the $\lambda$-theory $\psi=\theta_{\phi}(M, P)$ is consistent and

$$
M_{i} \neq_{\psi} N_{i}, \text { for every } i \leq k .
$$

Then the following theorems are corollaries of the algebraic incompleteness theorem.

Theorem 19. Let $\phi$ be an r.e. $\lambda$-theory. Then, the interval $\mathrm{I}\left[\phi, \nabla\left[\right.\right.$ contains a subinterval $\mathrm{I}\left[\psi_{1}, \psi_{2}\right]$ satisfying the following conditions:

- $\psi_{1}$ and $\psi_{2}$ are distinct r.e. $\lambda$-theories,

- every $\psi \in \mathrm{I}\left[\psi_{1}, \psi_{2}\right]$ is omitted by the indecomposable semantics,

$-\operatorname{card}\left(\mathrm{I}\left[\psi_{1}, \psi_{2}\right]\right)=2^{\aleph_{0}}$.

Proof. Since $\phi$ is r.e. we know by [?, Prop. 17.1.9] that there exists a $\lambda$-term $Q$ such that $\theta_{\phi}(Q, M)$ is consistent for all $M \in \Lambda^{o}$. Note that, in particular, this implies $Q \neq_{\phi} \lambda a b . a$ and $Q \neq_{\phi} \lambda a b . b$.

Let $\psi_{1}=\theta_{\phi}(Q, \lambda a b . a) \wedge \theta_{\phi}(Q, \lambda a b . b)$. Obviously, the $\lambda$-theory $\psi_{1}$ is consistent, r.e. and contains $\phi$. By Lemma ??, $[Q]_{\psi_{1}}$ is a non-trivial central element of $\boldsymbol{\Lambda}_{\psi_{1}}$.

We apply Lemma ?? to the r.e. $\lambda$-theory $\psi_{1}$ and to the pairs $(Q, \lambda a b . a)$ and $(Q, \lambda a b . b)$ such that $Q \neq_{\phi} \lambda a b . a$ and $Q \neq_{\phi} \lambda a b . b$. We get a $\lambda$-term $R \in \Lambda^{o}$ such that $Q \neq_{\theta_{\psi_{1}}(R, P)} \lambda a b . a$ and 
$Q \neq_{\theta_{\psi_{1}}(R, P)} \lambda a b . b$, for all $\lambda$-terms $P \in \Lambda^{o}$. Let $\psi_{2}=\theta_{\psi_{1}}(R, \lambda a . a)$. We have that $\psi_{2}$ is a proper extension of $\psi_{1}$.

The term algebra $\boldsymbol{\Lambda}_{\psi_{2}}$ of $\psi_{2}$ is a homomorphic image of the term algebra $\boldsymbol{\Lambda}_{\psi_{1}}$ of $\psi_{1}$, then every equation satisfied by $\boldsymbol{\Lambda}_{\psi_{1}}$ is also satisfied by $\boldsymbol{\Lambda}_{\psi_{2}}$. In particular, the equations expressing that $Q$ is a central element. Finally, $[Q]_{\psi_{2}}$ is non-trivial as a central element because $Q \neq_{\psi_{2}} \lambda a b . a$ and $Q \neq \psi_{2} \lambda a b . b$.

Hence, for every $\lambda$-theory $\psi$ such that $\psi_{1} \subseteq \psi \subseteq \psi_{2}$ the equivalence class of $Q$ is a nontrivial central element of the term algebra of $\psi$.

We get the conclusion of the theorem because $\operatorname{card}\left(\mathrm{I}\left[\psi_{1}, \psi_{2}\right]\right)=2^{\aleph_{0}}$ by Thm. ??(iv).

Remark 4. From Lemma ?? it follows that all the $\lambda$-models $\mathbf{C}$ such that $\operatorname{Th}(\mathbf{C})$ belongs to the interval $\mathrm{I}\left[\psi_{1}, \psi_{2}\right]$ above, are directly decomposable.

Theorem 20. Let $\mathbb{C}_{\mathbb{D E C}}$ be the class of all directly decomposable $\lambda$-models. Then we have that

(i) $T h\left(\mathbb{C}_{\mathbb{D E C}}\right)$ has an antichain of cardinality $2^{\aleph_{0}}$.

(ii) $T h\left(\mathbb{C}_{\mathbb{D E C}}\right)$ contains countably many "pairwise incompatible" intervals of cardinality $2^{\aleph_{0}}$.

Proof. ( $i)$ Let $U_{n} \equiv \Omega\left(\lambda x_{1} \ldots x_{n} x . x\right)$ and $\underline{k}$ be the $k$-th Church's numeral. Given a permutation $\sigma$ of the set of Church's numerals, we write $\psi_{\sigma}, \phi_{\sigma}$ for the $\lambda$-theories respectively generated by:

$$
\begin{aligned}
& E_{\sigma}^{1}=\left\{U_{0}=\lambda a b . a\right\} \cup\left\{U_{n}=\sigma(\underline{n-1}): n \geq 1\right\}, \\
& E_{\sigma}^{0}=\left\{U_{0}=\lambda a b . b\right\} \cup\left\{U_{n}=\sigma(\underline{n-1}): n \geq 1\right\} .
\end{aligned}
$$

From [?, Thm. 22] we get that $\psi_{\sigma}, \phi_{\sigma}$ are consistent and hence we have that the equivalence class of $U_{0}$ is a non-trivial central element of $\boldsymbol{\Lambda}_{\psi_{\sigma} \wedge \phi_{\sigma}}$. Thus, $\psi_{\sigma} \wedge \phi_{\sigma} \in T h\left(\mathbb{C}_{\mathbb{D E C}}\right)$ by Lemma ??.

If $\sigma_{1}, \sigma_{2}$ are two distinct permutations of the set of Church's numerals, then $\psi_{\sigma_{1}} \wedge \phi_{\sigma_{1}}$ and $\psi_{\sigma_{2}} \wedge \phi_{\sigma_{2}}$ are incompatible, because it is inconsistent to equate $\underline{n}=\underline{m}$ for every $n \neq m$.

Hence, $(i)$ follows since there exist $2^{\aleph_{0}}$ permutations $\sigma$ of the set of Church's numerals which give rise to pairwise incompatible $\lambda$-theories $\psi_{\sigma} \wedge \phi_{\sigma} \in T h\left(\mathbb{C}_{\mathbb{D E C}}\right)$.

(ii) Let $\sigma$ be a permutation of the Church's numerals and $\psi_{\sigma}, \phi_{\sigma}$ be as in the proof of $(i)$. Suppose that $\sigma$ is computable, then both $\psi_{\sigma}$ and $\phi_{\sigma}$ are r.e. $\lambda$-theories, hence also $\psi_{\sigma} \wedge \phi_{\sigma} \in$ $\operatorname{Th}\left(\mathbb{C}_{\mathbb{D E C}}\right)$ is r.e. Thus, the interval $\mathrm{I}\left[\psi_{\sigma} \wedge \phi_{\sigma}, \nabla\right]$ contains an interval of $2^{\aleph_{0}} \lambda$-theories belonging to $T h\left(\mathbb{C}_{\mathbb{D E C}}\right)$. The theorem follows since there exist countably many computable permutations $\sigma$.

Corollary 4. The indecomposable semantics, and hence the Scott-continuous, the stable and the strongly stable semantics omit a set of $\lambda$-theories which has an antichain of cardinality $2^{\aleph_{0}}$, and even contains countably many pairwise incompatible intervals of cardinality $2^{\aleph_{0}}$.

\section{Open problems}

In this section we collect open problems and conjectures that are related to universal algebra and topology. We start with the lattice of $\lambda$-theories.

\subsection{The lattice of $\lambda$-theories}

At the end of the nineties, the second author proposed the following conjecture: 
(P1) The lattice $\lambda T$ satisfies no (non-trivial) lattice identity.

This conjecture is still open. The best we know about this problem was shown in [?]: for any non-trivial lattice identity $e$, there exists a natural number $n$ such that the identity $e$ fails in the lattice of $\lambda$-theories over a language of lambda calculus extended with $n$ constants.

Another interesting problem to investigate is related to the lattices that are embeddable into $\lambda T$ and in the congruence lattices of $\lambda$-abstraction algebras. In [?] it has recently been shown that every finite Boolean lattice can be embedded into $\lambda T$ at the top. We propose the following conjecture:

(P2) Every finite lattice can be embedded into $\lambda T$.

Recall from [?] that the non-modular pentagon $N_{5}$ is a sublattice of $\lambda T$ and that by Visser [?] every countable partially ordered set embeds into $\lambda T$ by an order-preserving map. It is not difficult to prove that the class $L(\mathrm{LAA})$ of lattices embeddable into the congruence lattices of $\lambda$-abstraction algebras is a prevariety (i.e., it is closed under isomorphism, subalgebras and direct products). We conjecture that

(P3) $L(\mathrm{LAA})$ is the variety of all lattices.

Meet irreducible elements give important information on the structure of a lattice. Then, it is natural to investigate what $\lambda$-theories are meet irreducible. We have the following conjecture:

(P4) The least $\lambda$-theory $\lambda \beta$ is meet irreducible.

Other interesting problems arise when we classify the $\lambda$-theories as sensible, semisensible and non-semisensible. We recall that $\mathcal{H}^{*}$ is the unique maximal consistent sensible $\lambda$-theory.

(P5) What are the properties of the function mapping a $\lambda$-theory $\phi$ into the maximal semisensible theory $\phi \wedge \mathcal{H}^{*}$ contained within $\phi$ ?

From Prop. ?? we have that $\phi \wedge \mathcal{H}^{*}=\lambda \beta$ iff $\phi=\lambda \beta$.

Another problem is related to the equations between non- $\beta$-equivalent $\lambda$-terms which are consistent with every $\lambda$-theory. Their existence is a consequence of a result by Statman [?] stating that the meet of all coatomic $\lambda$-theories is not $\lambda \beta$.

(P6) Classify the identities consistent with every $\lambda$-theory

Other problems are related to the commutator:

(P7) Define non-trivial $\lambda$-theories $\phi$ and $\psi$ such that the commutator $[\phi, \psi]$ is strictly under $\phi \wedge \psi$.

These $\lambda$-theories must exist, because the following basic property of commutator

$$
[\phi, \psi]=[\gamma, \psi]=\delta \Rightarrow[\phi \vee \gamma, \psi]=\delta
$$

would imply the meet semidistributivity:

$$
\phi \wedge \psi=\gamma \wedge \psi=\delta \Rightarrow(\phi \vee \gamma) \wedge \psi=\delta,
$$

and this property does not hold in $\lambda T$.

The following are other interesting questions: 
(P8) What varieties of $\lambda$-abstraction algebras have a "good" commutator?

(P9) Is there a property of the commutator which holds for $\lambda$-theories but not for $\lambda$-abstraction algebras?

All the known properties of the commutator for $\lambda$-theories (see Section ?? and [?]) are also true for 0,1-algebras, i.e., algebras having a binary term with a right unit and a right zero. Then it is natural to rise the following question:

(P10) Is it possible to find a property of the commutator which distinguish 0,1 -algebras and $\lambda$ abstraction algebras or, more generally, find a new commutator distinguishing 0, 1-algebras and $\lambda$-abstraction algebras in a natural way?

\subsection{Models of lambda calculus}

Concerning the models of $\lambda$-calculus, the result of incompleteness stating that any semantics given in terms of partial orderings with a bottom element is incomplete leads us to the following problem.

(P11) Find a class of models of lambda calculus, where partial orders and Scott topology do not play any role.

As for the lattice of $\lambda$-theories, results on the structure of the set of $\lambda$-theories induced by a semantics are still rare, and there exist several longstanding basic open questions. The following natural questions were raised by Berline [?]:

(P12) Given a class of models of lambda calculus, is there a least $\lambda$-theory represented in the class?

(P13) Given a class of models of lambda calculus, is there a least sensible $\lambda$-theory represented in the class?

These two problems are related to one of the longstanding open problems of lambda calculus raised by Honsell and Ronchi della Rocca [?]:

(P14) Is there a "non-syntactical" model of the untyped lambda calculus whose theory is exactly the least (extensional) $\lambda$-theory $\lambda \beta(\lambda \beta \eta)$ ?

Di Gianantonio, Honsell and Plotkin [?] have shown that there exists an extensional $\lambda$-theory which is minimal among those represented by Scott continuous semantics.

Graph models and other classes of models. Graph semantics is the semantics $\mathcal{G}$ of lambda calculus given in terms of graph models. The reasons to concentrate on $\mathcal{G}$ are the following. $\mathcal{G}$ is, by far, the simplest class of models, nevertheless it contains a continuum of models with distinct theories, so it is a rich class. Moreover, the techniques and results for $\mathcal{G}$ can often be transferred to other classes of models.

Bucciarelli and Salibra [?,?,?] have shown that graph semantics admits a least graph theory (where "graph theory" means " $\lambda$-theory of a graph model") and a least sensible graph theory. The least graph theory is not equal to $\lambda \beta$ and it is trivially different from $\lambda \beta \eta$. The following interesting and difficult question is open: 
(P15) Is the least sensible graph theory equal to the $\lambda$-theory $\mathcal{H}$ generated by equating all unsolvable terms?

In [?] it was also shown that the $\lambda$-theory $\mathcal{B}$ (generated by equating $\lambda$-terms with the same Böhm tree) is the greatest sensible graph theory. This result is a consequence of the fact that the graph semantics omits all equations $M=N$ between $\lambda$-terms which do not have the same Böhm tree, but have the same Böhm tree up to (possibly infinite) $\eta$-equivalence.

(P16) What are the equations omitted by the other semantics of lambda calculus (i.e., filter models, stable models,...)?

In [?] it was recently shown that any "effective" model $\mathcal{D}$ of lambda calculus has an ordertheory (i.e., $\left\{M \leq N:|M| \sqsubseteq_{\mathcal{D}}|N|\right\}$ ) which is not r.e., so that $\lambda \beta$ and $\lambda \beta \eta$ cannot be theories of effective models. This enough surprising result holds in a strong way for graph models: the least graph theory is the theory of an effective graph model and the order-theory of every graph model is not r.e.

The following open problems deserve to be deeply investigated:

(P17) Is there a least "filter theory" (where "filter theory" means " $\lambda$-theory of a filter model" [?])? If yes, is there an effective model representing it?

(P18) Is there a least sensible filter theory?

\subsection{The order-incompleteness problem}

One of the most interesting open problems of lambda calculus is whether every $\lambda$-theory arises as the equational theory of a non-trivially ordered model (in other words, whether the semantics of lambda calculus given in terms of non-trivially ordered models is complete). Selinger [?] gave a syntactical characterization of the order-incomplete $\lambda$-theories (i.e., the theories not induced by any non-trivially ordered model) in terms of so-called generalized Mal'cev operators. In an algebraic setting the problem of the order-incompleteness can be expressed as follows:

(P19) Is there an n-permutable variety of $\lambda$-abstraction algebras for some $n \geq 2$ (see [?] for the definition of n-permutability)?

Plotkin, Selinger and Simpson [?] have shown that there exists no 2-permutable variety of $\lambda$ abstraction algebras and no 3-permutable variety of $\lambda$-abstraction algebras. It is open the case $n \geq 4$.

Selinger has shown in [?] that the problem of the order-incompleteness is also related to the following question by Plotkin [?]:

(P20) Is there an absolutely unorderable combinatory algebra, i.e., a combinatory algebra which cannot be embedded in any non-trivially partially ordered combinatory algebra?

\subsection{Topology and lambda calculus}

Scott topology is at the center of Scott continuous semantics and its refinements. In [?] it was shown that the semantic of lambda calculus given in terms of topological metric spaces is complete. Then it is natural to investigate the following problems:

(P21) Are there topological models of lambda calculus with a significant topology different from Scott topology? 
(P22) Are there other (i.e. different from metric space) classes of topological models which are complete semantics of the lambda calculus?

We recall that many authors tried to find models in Cartesian closed categories of topological spaces. Abramsky (see [?, Thm. 5.11]) and Plotkin (see [?, Thm. 5.14]) have shown respectively that there exists no non-degenerate model of the lambda calculus in the category of posets and monotone mappings, and in the category of complete ultrametric spaces and non-expansive mappings. Hoffmann and Mislove [?] have shown that the category of $k$-spaces and continuous maps has no non-degenerate, compact $T_{2}$-topological model. A $k$-space is a topological space in which a subset is open if and only if its intersection with each compact subset of the space is open in the subspace. The following problem is still open.

(P23) (Hoffmann-Mislove) Is there a model of lambda calculus in the category of $k$-spaces?

Notice that every topological model, in which all continuous selfmaps of the model are representable (as in the category of $k$-spaces), must have a connected topology because of the existence of fixed points. Then the following natural question arises:

(P24) Is the semantics of lambda calculus given in terms of connected topological models, complete?

Orderability/Unorderability. Selinger [?] has shown that the term algebras of the $\lambda$-theories $\lambda \beta$ and $\lambda \beta \eta$ are unorderable (i.e., they do not admit a non-trivial compatible partial order). Salibra [?] has found out a continuum of $\lambda$-theories whose term algebras are unorderable.

The classification of the $\lambda$-models into orderable/unorderable models can be refined as follows (see [?]). For every $\lambda$-model $\mathbf{C}$, let $T_{i}^{\mathbf{C}}\left(i=0,1,2,2_{1 / 2}\right)$ be the set of all topologies $\tau$ on $C$ which make $(\mathbf{C}, \tau)$ a $T_{i}$-topological model, where $T_{0}, \ldots, T_{2_{1 / 2}}$ are the usual topological separation axioms. It is obvious that in general we have

$$
T_{0}^{\mathbf{C}} \supseteq T_{1}^{\mathbf{C}} \supseteq T_{2}^{\mathbf{C}} \supseteq T_{2_{1 / 2}}^{\mathbf{C}} .
$$

We recall that a topology with a non-trivial specialization order (i.e., such that $a<b$ for some $a, b)$ would be $T_{0}$ but not $T_{1}$, so that

$$
\mathbf{C} \text { is unorderable iff } T_{0}^{\mathbf{C}}=T_{1}^{\mathbf{C}} .
$$

We say that a $\lambda$-theory $\phi$ is of (topological) type $i\left(i=0,1,2,2_{1 / 2}\right)$ if the term algebra of $\phi$ satisfies $T_{0}^{\boldsymbol{\Lambda}_{\phi}}=T_{i}^{\boldsymbol{\Lambda}_{\phi}}$. All $\lambda$-theories are of type 0 ; the $\lambda$-theory $\mathcal{B}$, generated by equating two $\lambda$-terms if they have the same Böhm tree, is not of type 1 (see [?]). $\lambda \beta$ and $\lambda \beta \eta$ are of type 1 by Selinger's result, while the $\lambda$-theory $\Pi$ found out by Salibra in [?] is of type $2_{1 / 2}$. Then the following natural question arises:

(P25) Is $\lambda \beta(\lambda \beta \eta)$ of type 2 ?

(P26) Is $\lambda \beta(\lambda \beta \eta)$ of type $2_{1 / 2}$ ?

\section{Conclusions and further works}

We generalized the Stone representation theorem to combinatory and $\lambda$-abstraction algebras showing that every combinatory and $\lambda$-abstraction algebra can be decomposed as a weak product 
of directly indecomposable algebras. We showed that the semantics of $\lambda$-calculus given in terms of directly indecomposable $\lambda$-models, although huge enough to include all the main semantics, is hugely incomplete. This gives a strong, uniform and elegant proof of the incompleteness of the continuous, stable and strongly-stable semantics.

A related question is whether there exists a notion of decomposition which respects the partial ordering of a model. Indeed there is no reason why the decomposition operators introduced in this paper should decompose the $\lambda$-model respecting the associated ordering. Hence, it would be interesting to find new kinds of decompositions which take into account also the partial order. On the other hand, the result of incompleteness in [?], stating that any semantics of $\lambda$-calculus given in terms of partial orderings with a bottom element is incomplete, removed the belief that partial orderings were intrinsic to $\lambda$-models. It is an open problem to find new Cartesian closed categories, where the partial orderings play no role and where the reflexive objects are directly indecomposable as combinatory algebras.

\section{References}

1. S. Abramsky. Domain theory in logical form. Annals of Pure and Applied Logic, 51:1-77, 1991.

2. J. Baeten and B. Boerboom. Omega can be anything it should not be. Indag. Mathematicae, 41:111-120, 1979.

3. H.P. Barendregt. Some extensional term models for combinatory logics and $\lambda$-calculi, Dissertation, University of Utrecht, 1971.

4. H.P. Barendregt. The lambda calculus: Its syntax and semantics. North-Holland Publishing Co., Amsterdam, 1984.

5. H.P. Barendregt, M. Coppo and M. Dezani-Ciancaglini. A filter lambda model and the completeness of type assignment. J. Symbolic Logic, 48:931-940, 1984.

6. O. Bastonero and X. Gouy. Strong stability and the incompleteness of stable models of $\lambda$-calculus. Annals of Pure and Applied Logic, 100:247-277, 1999.

7. A. Berarducci. Infinite $\lambda$-calculus and non-sensible models, in: P.Agliano, A.Ursini (Eds.), Logic and Algebra, Marcel-Dekker, NewYork, 1996.

8. C. Berline. From computation to foundations via functions and application: The $\lambda$-calculus and its webbed models. Theoretical Computer Science, 249:81-161, 2000.

9. C. Berline. Graph models of $\lambda$-calculus at work, and variations. Math. Struct. in Comp. Science, 16:185$221,2006$.

10. C. Berline and A. Salibra. Easiness in graph models, Theoretical Computer Science 354:4-23, 2006.

11. C. Berline, G. Manzonetto and A. Salibra. Lambda theories of effective lambda models, Proc. of 16th EACSL Annual Conference on Computer Science and Logic (CSL'07), LNCS 4646, pp. 268-282, Springer-Verlag, 2007.

12. G. Berry. Stable models of typed lambda-calculi. In Proc. 5th Int. Coll. on Automata, Languages and Programming, LNCS 62, Springer-Verlag, Berlin, 1978.

13. D. Bigelow and S. Burris. Boolean algebras of factor congruences. Acta Sci. Math., 54:11-20, 1990.

14. G. Birkhoff. On the structure of abstract algebras. Proc. Cambridge Philos. Soc., 31:433-454, 1935.

15. C. Böhm. Alcune proprietá delle forme $\beta$ - $\eta$-normali nel $\lambda$ - $K$-calcolo. Pubblicazioni dell'Istituto per le Applicazioni del Calcolo n. 696, Roma, 1968.

16. A. Bucciarelli and T. Ehrhard. Sequentiality and strong stability. Sixth Annual IEEE Symposium on Logic in Computer Science, IEEE Computer Society Press, 138-145, 1991.

17. A. Bucciarelli and A. Salibra. The minimal graph model of lambda calculus. 28th International Symposium on Mathematical Foundations of Computer Science, LNCS 2747, Springer-Verlag, 2003.

18. A. Bucciarelli and A. Salibra. The sensible graph theories of lambda calculus. 19th Annual IEEE Symposium on Logic in Computer Science (LICS 2004), IEEE Computer Society Press, 2004. 
19. A. Bucciarelli and A. Salibra. Graph lambda theories. Mathematical Structures in Computer Science, 2008. (to appear).

20. A. Church. A set of postulates for the foundation of logic. Annals of Math., 2:346-366, 1933.

21. A. Church. The calculi of lambda conversion. Princeton University Press, Princeton, 1941.

22. S. Burris and H.P. Sankappanavar. A course in universal algebra. Springer-Verlag, Berlin, 1981.

23. S. Burris and H. Werner. Sheaf constructions and their elementary properties. Trans. Amer. Math. Soc. 248:269-309, 1979.

24. A. Church. A set of postulates for the foundation of logic. Annals of Math., 2:346-366, 1933.

25. S. Comer. Representations by algebras of sections over boolean spaces. Pacific J. Math., 38:29-38, 1971.

26. M. Coppo and M. Dezani. An extension of the basic functionality theory for the $\lambda$-calculus. Notre Dame J. Formal Logic, 21:685-693, 1980.

27. M. Coppo, M. Dezani, F. Honsell and G. Longo. Extended type structures and filter lambda models. Logic Colloquium'82, Elsevier Science Publishiers, pp. 241-262, 1984.

28. H.B. Curry and R. Feys. Combinatory logic, Vol. I. North-Holland, Amsterdam, 1958.

29. M. Dezani-Ciancaglini, P. Severi and F.J. de Vries. Infinitary Lambda Calculus and Discrimination of Berarducci Trees, Theoretical Computer Science, 298(2):275-302, 2003.

30. P. Di Gianantonio and F. Honsell. An abstract notion of application. Typed lambda calculi and applications, LNCS 664, Springer-Verlag, 124-138, 1993.

31. P. Di Gianantonio, F. Honsell and G.D. Plotkin. Uncountable limits and the lambda calculus. Nordic J. Comput., 2:126-145, 1995.

32. V. Diercks, M. Erné and J. Reinhold. Complements in lattices of varieties and equational theories. Algebra Universalis, 31(4):506-515, 1994.

33. E. Engeler. Algebras and combinators. Algebra Universalis 13(3): 389-392, 1981.

34. R. Freese and R. McKenzie. Commutator theory for congruence modular varieties, Cambridge University Press, Cambridge, 1987.

35. H.P. Gumm. Geometrical methods in congruence modular algebras, Memoirs of the American Mathematical Society, 1983.

36. X. Gouy. Etude des théories équationnelles et des propriétés algébriques des modéles stables du $\lambda$ calcul. Thèse, Université de Paris 7, 1995.

37. J. Hagemann and C. Herrmann. A concrete ideal multiplication for algebraic systems and its relation to congruence distributivity, Arch. Math. 32: 234-245, 1979.

38. K.H. Hofmann and M.W. Mislove. All compact Hausdorff lambda models are degenerate. Fundamenta Informaticae, 22: 23-52, 1995.

39. F. Honsell and S. Ronchi della Rocca. An approximation theorem for topological lambda models and the topological incompleteness of lambda calculus. Journal of Computer and System Sciences 45:49-75, 1992.

40. P.T. Johnstone. Stone spaces. Cambridge University Press, 1982.

41. K. Kearnes and A. Szendrei, The relationship between two commutators, International Journal of Algebra and Computation, 8:497-531, 1998.

42. J.L. Kelley. General topology. D. van Nostrand Company, 1955.

43. J.R.Kennaway, J.W. Klop, M.R. Sleep and F.J. de Vries. Infinitary lambda calculus, Theoret. Comput. Sci. 175:93-125, 1997.

44. R. Kerth. Isomorphisme et équivalence équationnelle entre modèles $d u$-calcul. Thèse, Université de Paris 7, 1995.

45. R. Kerth. Isomorphism and equational equivalence of continuous lambda models. Studia Logica, 61:403-415, 1998.

46. R. Kerth. On the construction of stable models of $\lambda$-calculus. Theoretical Computer Science, 269:2346, 2001.

47. Lambda-calcul types et modéles, Masson, 1990.

48. W. A. Lampe. A property of the lattice of equational theories. Algebra Universalis, 23: 61-69, 1986. 
49. P. Lipparini. Commutator theory without join-distributivity. Transactions of the American Mathematical Society, 346:177-202, 1994.

50. P. Lipparini. A characterization of varieties with a difference term, II: neutral = meet semidistributivity. Canad. Math. Bull. 41:318-327, 1998.

51. S. Lusin and A. Salibra. The lattice of lambda theories. Journal of Logic and Computation, 14:373-394, 2004.

52. G. Manzonetto and A. Salibra. Boolean algebras for lambda calculus. 21th Annual IEEE Symposium on Logic in Computer Science (LICS 2006), IEEE Computer Society Press, 2006.

53. G. Manzonetto and A. Salibra. From lambda calculus to universal algebra and back. 33rd International Symposium on Mathematical Foundations of Computer Science, LNCS, Springer-Verlag, 2008.

54. R.N. McKenzie, G.F. McNulty and W.F. Taylor. Algebras, Lattices, Varieties, Volume I. Wadsworth Brooks, Monterey, California, 1987.

55. A.R. Meyer. What is a model of the lambda calculus?, Information and Control. 52:87-122, 1982.

56. M.W. Mislove. Topology, domain theory and theoretical computer science. Topology and its Applications, 89: 3-59, 1998.

57. R.S. Pierce. Modules over commutative regular rings. Memoirs Amer. Math. Soc., 1967.

58. D. Pigozzi and A. Salibra. An introduction to lambda abstraction algebras. IX Simposio Latinoamericano de Logica Matematica 38. Notas de Logica Matematica Universidad Nacional del Sur, Bahia Blanca, Argentina, 93-111, 1993.

59. D. Pigozzi and A. Salibra. Lambda abstraction algebras: representation theorems. Theoretical Computer Science, 140:5-52, 1995.

60. D. Pigozzi and A. Salibra. Lambda abstraction algebras: coordinatizing models of lambda calculus. Fundam. Inf., 33(2):149-200, 1998.

61. G.D. Plotkin. Set-theoretical and other elementary models of the $\lambda$-calculus. Theoretical Computer Science, 121:351-409, 1993.

62. A. Salibra and R. Goldblatt. A finite equational axiomatization of the functional algebras for the lambda calculus, Inform. and Comput., 148:71-130, 1999.

63. A. Salibra. On the algebraic models of lambda calculus. Theoretical Computer Science, 249:197-240, 2000.

64. A. Salibra. Nonmodularity results for lambda calculus. Fundamenta Informaticae, 45:379-392, 2001.

65. A. Salibra. A continuum of theories of lambda calculus without semantics. 16th Annual IEEE Symposium on Logic in Computer Science (LICS 2001), IEEE Computer Society Press, 334-343, 2001.

66. A. Salibra. Topological incompleteness and order incompleteness of the lambda calculus. (LICS'01 Special Issue). ACM Transactions on Computational Logic, 4:379-401, 2003.

67. D.S. Scott. Models for the $\lambda$-calculus. Manuscript (unpublished), 53pp., 1969. In Toposes, Algebraic geometry and Logic. LNM 274, Springer-Verlag, Berlin, 1972.

68. D.S. Scott. Continuous lattices. In Toposes, Algebraic geometry and Logic. LNM 274, Springer-Verlag, Berlin, 1972.

69. D.S. Scott. Data types as lattices. SIAM J. Comput., 5: 522-587, 1976.

70. Scott, D.S., Lambda calculus: some models, some philosophy, The Kleene Symposium, North-Holland, Amsterdam, 1980.

71. P. Selinger. Order-incompleteness and finite lambda reduction models. Theoretical Computer Science, 309:43-63, 2003.

72. M. Schönfinkel, Uber die Bausteine der mathematischen Logik. Math. Ann., 92:305-316, 1924.

73. J. D. H. Smith, Mal'cev varieties, Lecture Notes in Mathematics 554, Springer-Verlag, Berlin, 1976.

74. Richard Statman. Marginalia to a Theorem of Jacopini. Fundam. Inform., 45(1-2): 117-121, 2001.

75. D. Vaggione. $\mathcal{V}$ with factorable congruences and $\mathcal{V}=I \Gamma^{a}\left(\mathcal{V}_{D I}\right)$ imply $\mathcal{V}$ is a discriminator variety. Acta Sci. Math., 62:359-368, 1996.

76. D. Vaggione. Varieties in which the Pierce stalks are directly indecomposable. Journal of Algebra, 184:424-434, 1996.

77. A. Visser. Numerations, $\lambda$-calculus and arithmetic. In: (J.R. Hindley and J.P. Seldin eds.) To H.B. Curry: Essays on Combinatory Logic, Lambda-Calculus and Formalism. Academic Press, New York, 259-284, 1980. 